\title{
Infrared-Image Classification Using Hidden Markov Trees
}

\author{
Priya Bharadwaj and Lawrence Carin \\ Department of Electrical and Computer Engineering \\ Duke University \\ Durham, NC 27708-0291
}

\begin{abstract}
Images of three-dimensional targets are characterized by the target subcomponents visible from a particular target-sensor orientation (target pose), with the image often changing quickly with variable pose. We define a class as a set of contiguous target-sensor orientations over which the associated target image is relatively stationary with aspect, and therefore each target is in general characterized by multiple classes. Our feature parser employs a distinct set of Wiener filters for each class of images, to identify the presence of target subcomponents. The Wiener filters are designed using a KarhunenLoeve expansion. The statistical relationships between the different target subcomponents are modeled via a hidden Markov tree (HMT). The HMT classifier is discussed and example results are presented for forward-looking-infrared (FLIR) imagery of several vehicles. Classification performance is compared with that of several other competing classifiers.
\end{abstract}

\section{Introduction}

Classification of images is a problem of long-standing interest, involving applications in various areas such as target identification, medical diagnosis, and character recognition. In this paper we propose a new image-classification technique, employing Wiener filters [1] and a hidden Markov tree [2] (HMT). We apply the HMT algorithm to the problem of determining the identity of vehicles, by processing twodimensional forward-looking infrared (FLIR) imagery. 
A FLIR image typically varies quickly as a function of target-sensor orientation (i.e., as a function of the target pose). As the target pose changes, particular components of the target become visible and others are hidden. We define a class of a given target as a set of target-sensor orientations over which the associated images remain relatively invariant or stationary. In the context of classification, each image under test is assigned to a particular class, and consequently by determining the class association we implicitly also ascertain the target identity.

Images can be classified by focusing on the target subcomponents visible in each class, and by considering the relationships between their relative positions and excitation strengths. We employ a set of templates for representation of the target subcomponents, with the templates designed via a Karhunen-Loeve (KL) expansion based on training data. Rather than employing direct matched filtering, the KL templates are used to design Wiener filters [1], with this approach referred to as expansion matching (EXM) filtering [1]. There is a set of EXM filters for each class-dependent set of target components, with the relative position of the filters dictated by the expected relative position of the target components. For $N$ target components, correlation of a test image with the associated component-dependent EXM filters yields an $N$-dimensional feature vector.

One of the challenges in the classification of FLIR images is that, for a fixed target pose, one can realize multiple images, with the ensemble of FLIR images accounting for variable target history and imaging conditions. For example, the FLIR signature of a vehicle depends on the temperature of the vehicle (e.g. how long the engine has been on or off), weather conditions, and on the characteristics of background clutter. If a subcomponent is occluded or is relatively cold in the FLIR image, the correlation with the corresponding EXM filter yields a low value. Conversely, if the entire subcomponent is present (not occluded) and is warm, the correlation value with the corresponding EXM filter is high. Therefore, a two-state model ("low" and "high" states) is used to model the statistical variation of each component of the feature vector (corresponding to a particular target subcomponent), with the statistics of each state modeled via a distinct Gaussian density [2,3]. Further, the states sampled by successive 
coefficients of the feature vector are modeled as a Markov process. The Markov model is motivated in part by Markov random fields (MRF) [4,5]. In [5] MRFs are used to characterize the interactions between the neighboring pixels for texture classification. In this paper we utilize a Markov model to characterize the relationship between neighboring target subcomponents (rather than pixels). The physical relationships between the different target subcomponents are modeled in a tree-like fashion, yielding a hidden Markov tree (HMT), analogous to that applied previously in the context of wavelet analysis [2].

We compare the performance of the EXM-HMT image classification algorithm against several recently developed techniques. Hidden Markov trees were first proposed in the context of wavelet analysis, where they were used to model the relationships between wavelet coefficients at different levels (or scales) [2]. In our formulation the two Markov states correspond to target subcomponents being imaged brightly or weakly in a given image ("high" and "low" states, respectively), while in the context of a wavelet decomposition the two Markov states correspond to a given wavelet coefficient being large or small $[2,3]$. The wavelet filters are not necessarily well matched to a given class of imagery, motivating the aforementioned use of a KL expansion to design filters appropriate for a given class of data. Nevertheless, it is of interest to address the performance of the EXM-HMT algorithm relative to the original wavelet-based HMT classifier [2]. Such a comparison is considered in Sec. IV.

As a second comparative study, we consider an alternative use of hidden Markov models (HMMs). In particular, a recent paper [6] has employed the moments of the Radon transform to design HMMs for the purpose of image classification. The features used in the Radon-transform HMM (RT-HMM), the moments of the Radon transform, are analogous to measuring the dimensions of the image along one-dimensional projections about the image center of mass. While the RT-HMM performs well in classifying images that are of distinct size, the algorithm has difficulty when the sizes of the images are approximately the same. 
As a final comparative algorithm, we consider learning vector quantization (LVQ) [7] to encode the feature vectors. In particular, we use the same KL-based EXM filters discussed above, to generate the $\mathrm{N}$-dimensional feature vector. However, instead of modeling this feature vector via the HMT, we perform LVQ-based quantization [7], this termed EXM-LVQ. By comparing EXM-LVQ with EXM-HMT, both employing the same feature vectors, we address the quality of the HMT model vis-à-vis the simpler LVQ approach.

The remainder of the paper is organized as follows. In Sec. II we describe the feature extraction process using expansion-matching (EXM) filters. We model the statistical variation of the feature vectors for each class of images via a hidden Markov tree (HMT), as described in Sec. III. In Sec. IV we briefly present the alternative algorithms, in particular the wavelet-based HMT, RT-HMM and EXM-LVQ. Example results using forward-looking infrared (FLIR) images of four vehicles are presented in Sec. V. Conclusions and ideas for extending the work are presented in Sec. VI.

\section{Feature Extraction}

\subsection{Feature templates}

Consider the $m$ th target class, this corresponding to a contiguous set of targetsensor orientations over which the image of a given target is approximately stationary. Assume that the image of this target class is composed of $P_{m}-1$ subcomponents, with this made more explicit below. We derive representative templates for each of the $P_{m^{-}} 1$ target subcomponents, as well as a separate template representative of the composite target image. We therefore employ a total of $P_{m}$ templates for a given target class, $P_{m}$ in general class dependent. As an example, we have $P_{m}=7$ templates for the image in Fig. 1: one corresponding to the entire image, as indicated in Fig. 1(a), and six subcomponents, as indicated in Fig. 1(b). The choice of the number of subcomponents, as in Fig. 1(b), is dependent on the characteristics of the images of a particular class. There is clearly flexibility in the choice of the parameter $P_{m}$ as well as in the choice of the specific target 
subcomponents. This aspect of the algorithm has not been optimized, since for the FLIR imagery considered here the subcomponents are relatively clearly defined by the different components of the vehicle.

Assume a total of $M$ classes (across all targets of interest) and that we have $N_{m}$ training images for class $m$. Moreover, assume that the $m^{\text {th }}$ class is characterized by $P_{m}$ regions, in the sense discussed above. It is essential that the $N_{m}$ training images belonging to a particular class be centered about the same reference point, and that they are oriented at the same reference angle. Since we are dealing with training data, for which the actual target position is typically known, this alignment is not difficult. In the context of testing, for which the actual target identity and orientation is unknown, such alignment is not as simple. However, in the context of our algorithm this issue concerning the testing phase is relatively straightforward, as discussed further below.

\subsection{EXM filter formulation}

From the training data associated with class $m$, we have $N_{m}$ realizations of each of the $P_{m}$ regions. A traditional technique in pattern matching would employ the $N_{m}$ training images for each of the $P_{m}$ regions to derive a region-dependent set of eigenvectors [8]. This representation can then be used in the context of a region-dependent KarhudenLoeve (KL) transform [8]. As we discuss in Sec. 2.3, we ultimately employ a KL representation, but not based on the original $N_{m}$ training images directly. In particular, we first form a set of $N_{m}$ Wiener filters [1,8] for each of the $P_{m}$ class-dependent regions (a distinct filter designed from each of the $N_{m}$ training images), with the KL decomposition performed on the Wiener filters rather than on the original data. A summary of why we have employed this approach is discussed below.

Matched filters [8] are commonly used to correlate a particular pattern or feature template with a given signal, which in our case is a two-dimensional image. For a given 
feature template the matched filter is an optimal filter in the sense that the SNR is maximized, with SNR defined as the ratio of the filter's response at the center of the pattern to the variance of the filter's response to white Gaussian noise. However, one of the drawbacks of a matched filter [1,9] is that the response off the center of the feature can be high (since the matched filter is optimized only with respect to the response at the center of the template). As a result the response has a broad peak, and it is difficult to locate the features in the image, especially if the image has several similar features close to each other. In our work we have several proximate target subcomponents.

Expansion matching (EXM) filters [1,9] alleviate the above limitation of a matched filter. The EXM filter formulation is the Wiener filter solution for restoring a delta function blurred by the feature template [1,9]. By applying the EXM filter to the image and restoring the delta function, we determine the location of the feature template. The amplitude of the restored delta function gives us the correlation of the filter with the image. It is shown in [1] that the EXM filter maximizes a criterion called discriminative SNR (DSNR), by seeking to minimize the off-center response of the filter. The EXM filters, therefore, generate sharp peaks, and enhance the localization of features in an image.

Consider an $N \times N$ discrete image $I(x, y)$, which includes an attenuated version of a feature template $\phi(x, y)$ located at $\left(x_{o}, y_{o}\right)$ with additive noise $\lambda(x, y)$. Correlation-based techniques use a filter $\theta(x, y)$ such that a maximum is generated at $\left(x_{o}, y_{o}\right)$. The filter response obtained by correlating $\theta(x, y)$ with $I(x, y)$ is given by

$$
\begin{aligned}
z(x, y) & =\theta(x, y) * I(x, y) \\
& =c_{\phi} \phi\left(x-x_{o}, y-y_{o}\right) * \theta(x, y)+\lambda(x, y) * \theta(x, y) \\
& =z_{\phi}(x, y)+z_{\lambda}(x, y)
\end{aligned}
$$


In the frequency domain the EXM filter (Wiener filter, with the feature template as the blurring function and the delta function as the function that is to be restored) is implemented as follows [8]

$\Theta_{E X M}\left(\varpi_{1}, \varpi_{2}\right)=\frac{\bar{\Phi}\left(\varpi_{1}, \varpi_{2}\right)}{\left|\Phi\left(\varpi_{1}, \bar{\varpi}_{2}\right)\right|^{2}+\frac{S_{\lambda \lambda}\left(\varpi_{1}, \varpi_{2}\right)}{S_{c c}\left(\varpi_{1}, \bar{\varpi}_{2}\right)}}$

The matched filter is represented as

$\Theta_{M A T C H}\left(\varpi_{1}, \varpi_{2}\right)=\frac{\bar{\Phi}\left(\varpi_{1}, \varpi_{2}\right) e^{-j\left(\varpi_{1} \cdot x_{o}+\varpi_{2} \cdot y_{o}\right)}}{S_{\lambda \lambda}\left(\varpi_{1}, \varpi_{2}\right)}$

$\bar{\Phi}\left(\varpi_{1}, \varpi_{2}\right)$ is the complex conjugate of the Fourier transform of the feature template $\phi(x, y)$. The $S_{\lambda \lambda}$ and $S_{c c}$ are the power spectral densities of the noise and the input sequence (delta function), respectively.

The DSNR is defined as [1]

$\mathrm{DSNR}=10 \log \frac{z^{2}\left(x_{o}, y_{o}\right)}{\sum_{i \neq x_{o}} \sum_{j \neq y_{o}}[z(i, j)]^{2}}$

Since DSNR provides some control over the off-peak response, the EXM filter response has a sharper peak. The matched filter on the other hand is optimized for maximum SNR, which is defined as 
$\mathrm{SNR}=10 \log \frac{\left[z_{\phi}\left(\boldsymbol{x}_{\boldsymbol{o}}, \boldsymbol{y}_{\boldsymbol{o}}\right)\right]^{2}}{\left[\frac{1}{N^{2}} \sum_{\boldsymbol{N}} \sum_{\boldsymbol{N}}\left[z_{\lambda}(\boldsymbol{i}, \boldsymbol{j})\right]^{2}\right]}$

The off-center response is not addressed by the SNR criterion, and the peaks in the response are broad, often leading to problems in locating the template in the image (especially when there are several proximate components). Assuming white noise, the ratio

$$
\frac{S_{\lambda \lambda}\left(\varpi_{1}, \varpi_{2}\right)}{S_{c c}\left(\varpi_{1}, \varpi_{2}\right)}=\frac{S_{\lambda \lambda}}{S_{c c}}
$$

By increasing the ratio $\frac{S_{\lambda \lambda}}{S_{c c}}$, the response of the EXM filter tends towards the matchedfilter response, with broader peaks, but more robust to noise. By setting the ratio parameter $\frac{S_{\lambda \lambda}}{S_{c c}}$ to zero, the EXM filter becomes a high pass filter, i.e., an edge detector, with a sharp peak in the filter response, but highly sensitive to noise. In practical applications, the value of the ratio parameter should be set to achieve a tradeoff between a sharp peak in the response of the filter and robustness to noise. In the example results reported in Sec. V, we have set $\frac{S_{\lambda \lambda}}{S_{c c}}=10$ (note also that each template has been normalized by the energy in the template).

In Fig. 2 we consider the matched filter and EXM filter, and their respective performance, for the original image in Fig. 1(a). The response of the EXM filter has a sharp peak (Fig. 2(c)), whereas the response of the matched filter has a broad peak (Fig. 2(d)). The EXM filter and the matched filter are shown in Figs. 2(a) and 2(b), respectively. 
As described in Sec. 2.1, there are $N_{m}$ training realizations for each of the $P_{m}$ regions of class $m$. Let $\Omega_{n}^{m, p}$ be the EXM filter derived from the $n^{\text {th }}$ training image belonging to class $m$, region $p$. The $N_{m}$ EXM filters associated with the $N_{m}$ training realizations of region $p$ for class $m$ are denoted as $\Sigma_{m, p}$, where

$$
\Sigma_{m, p}=\left[\Omega_{1}^{m, p}, \Omega_{2}^{m, p}, \ldots, \Omega_{N_{m}}^{m, p}\right], \quad 1 \leq m \leq M, 1 \leq p \leq P_{m}
$$

We use the Karhunen-Loeve transform, explained in the next section, to reduce the computational complexity of correlating the image with the $N_{m}$ filters of $\Sigma_{m, p}$ to form the feature vector.

\subsection{Eigendetectors using Karhunen-Loeve transform}

The Karhunen-Loeve (KL) transform is an efficient means of representing the $P_{m}$ class-dependent feature detectors $\left(\Sigma_{m, 1}, \Sigma_{m, 2}\right.$, etc. $)$ by a smaller set of elements, and at the same time maintaining the performance of the original set of filters with minimal error [8]. Consider a set of zero-mean filters given by

$\bar{\Omega}_{i}^{m, p}=\Omega_{i}^{m, p}-\frac{1}{N_{m}} \sum_{i=1}^{N_{m}} \Omega_{i}^{m, p}=\Omega_{i}^{m, p}-\mu^{m, p}$

$R_{\Omega \Omega}$ is the covariance matrix of the EXM filter set, and is defined as

$\left[R_{\Omega \Omega}\right]=\frac{1}{N_{m}} \sum_{i=1}^{N_{m}}\left(\Omega_{i}^{m, p}-\mu^{m, p}\right)\left(\Omega_{i}^{m, p}-\mu^{m, p}\right)^{T}$

where the superscript $T$ represents transpose. The eigenvectors of the covariance matrix form the basis vectors of the KL transform,

$\left[R_{\Omega \Omega}\right] \phi_{i}^{m, p}=\eta_{i}^{m, p} \phi_{i}^{m, p}$ 
Thus, the KL transform produces an orthonormal set of basis functions $\phi_{i}^{m, p}$ for the given set of EXM filters. Note that we have chosen to perform the KL transform after designing the $N_{m}$ EXM filters, since the resulting set of eigenvectors are orthonormal. By contrast, if we were to first perform a KL transform of the training data and then produce EXM filters using the resulting eigenvectors, the resulting EXM will in general no longer be orthonormal.

The eigenvectors are arranged in the descending order of eigenvalues $\eta_{i}^{m, p}$; the mean square error (MSE) can be minimized by using the eigenvectors with the top $N_{\text {eig }}$ eigenvalues as a truncated basis to represent the entire filter set. The MSE error incurred by this truncation is represented by

$$
\begin{aligned}
& \text { Error }=\frac{1}{N_{m}} \sum_{i=1}^{N_{m}}\left\|\bar{\Omega}_{i}^{m, p}-\hat{\Omega}_{i}^{m, p}\right\|^{2}=\frac{1}{N_{m}} \sum_{i=1}^{N_{m}}\left\|\bar{\Omega}_{i}^{m, p}-\sum_{j=1}^{N_{e i g}} \alpha_{i j} \phi_{j}^{m, p}\right\|^{2} \\
& \alpha_{i j}=\bar{\Omega}_{i}^{m, p^{T}} \phi_{j}^{m, p}
\end{aligned}
$$

In general we choose $N_{e i g}<<N_{m}$. The set of $N_{\text {eig }}$ eigen templates corresponding to class $m$ and template $p$ is represented as $\mathrm{E}_{m, p}$

$$
\mathrm{E}_{m, p}=\left[\phi_{1}^{m, p}, \phi_{2}^{m, p}, \ldots, \phi_{N_{e i g}}^{m, p}\right], \quad 1 \leq m \leq M, 1 \leq p \leq P_{m}
$$

For each image class we have a set of eigen templates corresponding to each of the $P_{m^{-}}-1$ subcomponents of the FLIR image, and one set of eigen templates corresponding to the entire image. For notational purposes let $p=1$ denote the template corresponding to the entire image, while $p=2$ through $P_{m}$ denote the templates corresponding to the $P_{m^{-}}-1$ subcomponents in class $m$. The eigen template set $\mathrm{E}_{m, 1}$ is termed a coarse-feature eigen template, since it is derived from the entire target image, and $\mathrm{E}_{m, 2}$ to $\mathrm{E}_{m, P}$ are termed fine-feature eigen templates, since they are used to detect the 
presence of finer features or subcomponents of a target. It should be noted that $N_{\text {eig }}$ is not a fixed value, and that the value of $N_{\text {eig }}$ depends on the characteristics of the EXM filter set under consideration.

Figures 3 shows the energy extracted by the eigenvectors of the covariance matrix of $\Sigma_{m, 2}$ (note that $p=2$ corresponds to the first subcomponent) for subcomponent one in Fig. 1. The principal eigenvectors are employed to constitute the eigen EXM filters. In this example the top 50 eigenvectors $\left(N_{\text {eig }}=50\right)$ retain $90 \%$ of the total energy.

\subsection{Feature vector}

Each set of eigen templates is a compact representation of the variations in the corresponding target region, across the $N_{m}$ training realizations. In the testing phase, the feature vector is formed by correlating the image with the eigen templates of the EXM filters of each subcomponent of a particular class $m$, and determining the maximum value of the correlation in a localized neighborhood of the image (dictated by the subcomponent under consideration). Each such neighborhood is linked to the location of the corresponding target component in the image. We discuss this issue further below.

Consider training data from class $m$, composed of $N_{m}$ training images (as discussed above). The feature vector for image $n$ of this class is

$C_{m}^{n}=\left[\operatorname{corr}_{1}^{m, n}, \operatorname{corr}_{2}^{m, n}, \ldots, \operatorname{corr}_{P_{m}}^{m, n}\right], 1 \leq n \leq N_{m}$

where

$$
\operatorname{corr}_{p}^{m, n}=\max _{\text {neighborhood }}\left[\sum_{j=1}^{N_{\text {eip }}}\left|\frac{\left\langle I^{m, n}, \phi_{j}^{m, p}\right\rangle}{\left\langle I_{e x m}^{m, n}, I_{e x m}^{m, n}\right\rangle^{\frac{1}{2}}\left\langle\phi_{j}^{m, p}, \phi_{j}^{m, p}\right\rangle^{\frac{1}{2}}}\right|\right]
$$


$\left\langle I_{\text {exm }}^{m, n} I_{\text {exm }}^{m, n}\right\rangle$ is the energy in the image over the support of the filter and $I^{m, n}$ is the image under consideration. The feature vector in (13) is composed of the maximum values of correlation obtained by correlating the image with $P_{m}$ sets of eigen EXM templates. Each such value is denoted as $\operatorname{corr}_{p}^{m, n}$, where $p=1$ corresponds to the correlation with the coarse feature eigen templates, and $p=2$ to $p=P_{m}$ correspond to the correlation with the fine feature eigen teamplates.

Each of the $P_{m}$ regions associated with class $m$ corresponds to a physical location on the target, with known relative positioning (for the training data). Referring to (14), when computing the correlation for region $p$ we only search for the maximum correlation over a portion of the image consistent with that region (see Fig. 4). To define the appropriate portions of the image for definition of the $p$-dependent region, it is important to align the image appropriately. In this context, we first perform a correlation with eigen EXM filters associated with target component $p=1$, corresponding to the overall target shape. After finding the maximum correlation with this set of eigen EXM filters, the target image is repositioned in the center of the domain. After this alignment is performed (this particularly important for the testing data, for which alignment is unknown), the corresponding subcomponent regions are defined, in which we perform searches for the maximum correlations for regions $p=2$ through $p=P_{m}$. Figure 4 shows the neighborhood (indicated by the black squares in the image) for all the coefficients of the feature vector (13), for particular class $m$.

\section{Statistical Model: Hidden Markov Tree (HMT)}

In Sec. II we addressed extracting features from a given image, reducing the image to a feature vector $C_{m}^{n}$ as in (13). The value of each element of the feature vector (13), $\operatorname{corr}_{p}^{m, n}$, can be either "high" or "low" depending on the subcomponent excitation strength in the FLIR imagery. As indicated in the Introduction, this is connected to the temperature of the target component and whether the subcomponent is occluded. If a 
target subcomponent is excited strongly it is deemed in a "high" state, and otherwise it is in a "low" state. The states sampled by the coefficients of the feature vector are unknown, or "hidden" $[2,3]$. The statistics of the "high" and "low" states, corresponding to each element of the feature vector, are modeled via a distinct non-zero-mean Gaussian density (or, possibly, a Gaussian mixture, although such has been found unnecessary for the examples considered in Sec. V).

Having developed a two-state model for each coefficient, what remains is the manner in which these are interconnected. In [2], the HMT is used to interconnect the wavelet coefficients that are obtained by performing a $J$-level wavelet transform $[2,3]$ on the signal of interest. The wavelet transform is performed by decimating the signal at each level, resulting in the well-known multi-level tree structure of the wavelet coefficients. The wavelet coefficient at level $j$ is connected with two "children" coefficients at level $j-1[2,3]$.

The feature vectors of the images can be cast into a similar tree structure. Correlation with the eigen filters associated with $p=1$ (characteristic of the overall target shape), $\operatorname{corr}_{1}^{m, n}$, occupies the top of the tree (root node). Subsequent levels in the tree are formed by correlating the image with the fine eigen filters of the target's different subcomponents. Figure 5(a) shows the 3-level HMT used to classify the FLIR images considered in the examples considered below. Index $p$ in each node of the HMT, in Fig. 5(a), indicates that the node corresponds to $\operatorname{corr}_{p}^{m, n}$, with the associated subcomponent marked in Fig. 5(b). Each element of the image-classifier HMT is connected with two "children" at a lower level. Let $H$ and $L$ represent the "high" and "low" states of element $j+1$ of the HMT (see Fig. 5), with $H_{l}$ and $L_{l}$ similarly defined for node $j_{l}$. The transitions from node $j+1$ to the two children $j_{l}$ and $j_{r}$ are modeled as a Markov process, i.e., the state transitions to the "child" nodes are dependent only on the state of the "parent" node. There are four possible state transitions from $j+1$ to $j_{l}$ : element at $j+1$ could be $H$ and the element at $j_{l}$ could be $H_{l},\left[H, H_{l}\right]$; similarly we could have $\left[H, L_{l}\right],\left[L, H_{l}\right]$ or $\left[L, L_{l}\right]$. Each state transition, listed above, is characterized by a probability. A similar set of statetransition probabilities are defined between $j+1$ and $j_{r}$. The initial-state probability is 
defined as the probability that the top (root) node is in the "high" or "low" state. The hidden Markov tree is completely characterized by the dual-state model for each element (with the aforementioned single-Gaussian model applied per state), the state transition probabilities, and the initial state probability for the top node.

The binary tree structure in Fig. 5(a) is compatible with our understanding of the physical nature of infrared images. The intensity of infrared images is a function of the temperature of the vehicles. In such images, "high" and "low" states correspond to whether a particular part of the target is "hot" or "cold" (and/or whether it is occluded or not). Referring to Fig. 5, the states of nodes 2 and 3, i.e., correlation with the templates corresponding to the body of the vehicle, are dependent on the state of the correlation with the entire image, i.e. node 1 . If node 1 is in the "high" state, it means that the vehicle is predominantly "hot", and therefore, it is likely that nodes 2 and 3 are also in the "high" state. The states of nodes 2 and 3 are therefore modeled as being conditioned on the state of node 1 . Since parts 4 and 5 are close to 2 , the states of 4 and 5 are likewise modeled as being conditioned on the state of 2; similarly, the states of 6 and 7 are dependent on the state of 3 . The state of node 2 is, however, not exclusively dependent on state of node 1 . It may also be influenced by state of node 3, since both nodes 2 and 3 are connected via node 1 . Similarly, node 6 may be influenced by node 1 . It is also possible to model each subcomponent as being directly influenced by multiple neighboring subcomponents [10]. Such an approach, however, is not explored in this paper, and will be addressed in future work. It is also important to note that the form of the tree (definition of "parent" and "child") nodes is not unique and has not been optimized here.

In the training phase, an HMT is developed for each of the $M$ classes of images (recall that there are in general multiple classes per target). The testing phase involves classifying images as belonging to one of the $M$ classes. In the testing phase, we form feature vectors by correlating the image, of unknown class, with the eigen templates of each image class. The feature vectors are then passed through the respective classdependent HMTs, and the image is classified as belonging to the class for which the likelihood is maximum. 


\section{Comparative Algorithms}

\subsection{Radon transform HMM}

In the Radon-transform hidden-Markov-model (RT-HMM) algorithm [6], the Radon transform is employed to generate a sequence of one-dimensional projections of an image, at different angles about the image (Fig. 6), using angular sampling $\delta \theta$. The feature vector is comprised of $Q$ moments, and here we use $Q=3$ corresponding to variance, skewness and kurtosis, with such a feature vector computed for each of the onedimensional Radon projections. The sequence of associated feature vectors is classified via a hidden Markov model (HMM) [6,11-14]. As mentioned in the Introduction, the moments of the projections principally measure the dimensions and general shape of the image from different angles around the image. While this approach is successful in classifying images that are of different dimensions, the algorithm is not successful in classifying images of similar dimensions, as demonstrated in Sec. V.

We again have $M$ target classes (the same target classes are defined for each classifier), and a distinct RT-based HMM is developed for each class. The states of the RT-HMM (refer to [11-14]) are defined as the angular regions over which the Radon transform remains relatively unchanged, where here the states are defined in the context

of the angular position of the Radon transform. A Gaussian-mixture density is used to characterize the Q-dimensional feature vectors of each such state. In the testing phase, the feature vector of a given image is passed through each HMM, and it is classified as belonging to that model for which the conditional probability of the image given the model is maximum. In the examples in Sec. V we use four Gaussian mixtures per RTHMM state, with this found to yield the best performance for the data considered here. 


\subsection{Wavelet-based HMT}

The wavelet-HMT employs a quadtree structure [2], in which each parent node is connected to four child nodes, as shown in Fig. 7 (in the HMT model for the EXM-HMT algorithm discussed in Sec. III, we employ a binary tree structure). We here implement a decomposition based on the Haar wavelet, although the wavelet-based HMT is applicable to general wavelets. The wavelet decomposition of the FLIR images is performed to the coarsest level, and quadtree HMTs are developed for the sequence of high-high, high-low and low-high images, using the coarsest and two subsequent finer levels (a total of three levels). We go down to the coarsest wavelet level because to do otherwise leads to multiple quadtrees, and in general the imagery is spatially non-stationary. Consequently, for this case tying [3] is inappropriate. With the finite available imagery available for training, we cannot accurately estimate HMT parameters for more than three wavelet levels.

There is a wavelet quadtree for the sequence of high-high (HH), high-low (HL) and low-high (LH) FLIR imagery (for three levels), with these taken here as statistically independent, for simplicity. Therefore, we compute the total likelihood that a given image is associated with a given class as the product of the likelihoods of the three associated wavelet-quadtree HMTs. Due to variation in the pixel amplitudes of the image, caused by changing imaging conditions, the variance on "high" and "low" states of the wavelet coefficients is large. This vitiates the classification performance of the waveletHMT, for the data considered.

\subsection{LVQ using EXM features}

Learning vector quantization (LVQ) [7, 15], a supervised learning algorithm, has been applied to wavelet features [7] for classifiying FLIR images. In this paper we use LVQ on the features extracted via the EXM filters in (3), thereby employing a set of discrete codes to represent the image feature space. A codebook generated by vector quantization (VQ) [16] is used as the starting point for LVQ. The codebook is then 
refined by LVQ to maximize classification performance, translating into the separation between the class-dependent codebook elements (quantified by Euclidean distance metric). An image is classified as belonging to the same class as the code for which the Euclidean distance is minimum.

\section{Results}

\subsection{Methodology}

We employ the above algorithms to classify FLIR images of four vehicles. We observe that the images vary as a function of the target-sensor orientation, with the images formed at $5^{\circ}$ intervals around the vehicle. We identify four sets of angular regions (classes) for each vehicle, over which the images are relatively unchanged. Define $0^{\circ}$ as looking at the front end of the vehicle, as in Fig. 8. We define class 1 as comprised of images primarily from the front of the vehicle (angles $0-15^{\circ}, 345-360^{\circ}$ ), class 2 primarily comprises images of the sides of the vehicle (angle 20-160 ${ }^{\circ}$ ), class 3 primarily consists of images of the rear of the vehicle (angles $165-195^{\circ}$ ), and class 4 primarily consists of images of angles $200-340^{\circ}$. Since there are four classes for each vehicle, there are a total of $M=16$ classes. Example FLIR images are shown in Fig. 9, demonstrating the wide variability in the FLIR image for the same target pose (with this variability tied to the target history, i.e., how hot various portions of the target are). We also note the similarity of the FLIR images between different targets.

For each vehicle, class 1 and class 3 consist of 9 angles each (e.g., class 3 consists of angles $165-195^{\circ}$ at $5^{\circ}$ increments), while class 2 and class 4 consist of 27 angles each. For a given class, we used 9 images per angle for training and testing. For class 2 and class 4 of each vehicle we employ 243 images to train the HMT, and 243 distinct images are used for testing. For class 1 and class 3 of each vehicle we have employed 81 images for training and 81 distinct images for testing. We have $P_{m}=7$ templates for each image class: six target subcomponents and one for the entire image. It is not necessary, however, that all classes have the same number of templates, $P_{m}$, and the choice of the 
number of templates depends on the image class, as mentioned in Sec. II. We performed KLT on the training templates to generate a reduced set of eigen templates. All of the data considered are based on computational models that simulate the FLIR response of a vehicle, as a function of target history and pose. The data was generated by the US Army Research Laboratory, Adelphi, MD, USA.

We use the same set of training and testing images for all comparative algorithms: the RT-HMM, wavelet-HMT and EXM-LVQ. In the RT-HMM, we determine the Radon transform of the image at angular intervals of $\delta \theta=30^{\circ}$, as shown in Fig. 6. In the EXMLVQ we use 15 codes to characterize each class of images (a total of 15x16=240 codes).

Above we quantified the number of images of each class used for testing and training. In the context of examining algorithm performance, we varied the definition of which particular images were used for testing and training, to examine the statistical variability of the results. This issue is discussed further below.

\subsection{Analysis of results}

The classification results for the wavelet-HMT, RT-HMM, EXM-LVQ and EXMHMT algorithms are presented in Tables 1-4, respectively, in the form of confusion matrices. The diagonal elements of the confusion matrix indicate the percentage of correct classification for a particular class. The off-diagonal terms indicate the percentage of times a particular class of images was misclassified as another class. Since we have 16 classes, the confusion matrix should be a $16 \times 16$ matrix. Limitation in space precludes presentation of a $16 \times 16$ confusion matrix. However, as we mentioned earlier, classes 2 and 4 of each vehicle are approximately mirror images of each other, with the same holding for classes 1 and 3. While we train distinct HMTs for each of the four classes, we combine the (classification and misclassification) results of classes $2 \& 4$ and classes $1 \&$ 3 , and present the results (Tables 1-4) as $8 \times 8$ confusion matrices. 
In Fig. 10 we plot the number of images of a particular vehicle that have been misclassified by the EXM-HMT algorithm as belonging to the neighboring classes of the same vehicle. Results are shown here for vehicle one, with these representative of results from all four vehicles. These results show that the principal misclassifications between class $1 \& 3$ and class $2 \& 4$ (of the same vehicle) are on the boundaries, or transition regions between classes. Such a result is expected, since images in the transition regions display characteristic of both the adjoining classes, and hence it is difficult to classify these images as belonging to one or the other class. However, these images from the transition region between classes have been attributed to the correct vehicle.

We next present classification results for the four vehicles, with a correct classified defined if the correct vehicle is assigned, even if the associated target class is incorrect. In Tables 5-8 are shown results for the wavelet-HMT, RT-HMM, EXM-LVQ and EXM-HMT algorithms, respectively. The total average successful classification percentage for the EXM-HMT algorithm is $91 \%$. The wavelet-HMT algorithm achieves an average classification percentage of 73\%, the RT-HMM 71\%, and the EXM-LVQ 84\%. The above results are the mean (average) results computed using ten distinct mappings of the available FLIR imagery into training and testing bins. Of interest is the variability of these results, for which we quantify a standard deviation of $2.32 \%$ for EXM-HMT, $5.61 \%$ for EXM-LVQ, $1.2 \%$ for RT-HMM, and $2.44 \%$ for the waveletbased HMT.

\section{Conclusions and Future Work}

We have proposed an EXM-HMT algorithm for image classification. The algorithm has been applied to the problem of classifying vehicles via FLIR imagery. Since the images of a target vary as a function of the target-sensor angle, we segmented the target into classes (sets of contiguous target-sensor angles over which the FLIR imagery is approximately stationary), and developed an HMT for each target class. 
The feature parser employs eigen templates corresponding to the different visible subcomponents in a given target class. The subcomponents are linked to physical regions of the target. The key contribution of this paper involves development of a model that accounts for variations in the images due to changing target conditions. The HMT has been employed previously based on wavelet decomposition, although wavelet filters are not in general matched to the phenomenology characteristic of the FLIR imagery. The filters employed here are based on a Karhunen-Loeve decomposition generated via training data, with these linked to target subcomponents. We have also employed expansion-matching (EXM) for representation of the subcomponent templates, with such connected to a Wiener filter. We demonstrated significant improvement in classification performance of the EXM-HMT classifier, relative to its wavelet-HMT counterpart. The performance of the EXM-HMT was also shown to be markedly better than HMM classifier built around multiple Radon transforms of the image. Finally, in attempt to separate the issue of features used from the quality of the subsequent classifier, we also employed the EXM features in another classifier (other than the HMT). In particular, the EXM-based features were employed in the context of learning vector quantization (LVQ). The EXM-HMT classifier was markedly better than the EXM-LVQ, underscoring the quality of the HMT construct.

There are several topics worthy of further study. As has been discussed, in the context of the HMT representation of the EXM-filter-derived features, there are many ways in which to define the parent and children components in the tree. This is an issue that could be optimized to yield improved performance. Moreover, it is of interest to consider using multiple trees for a given target class, and fusing their outputs. The basic EXM-HMT algorithm could also be employed for the analysis of a sequence of FLIR images, in the context of video. In such a scenario one will typically view a given target from a sequence of classes (poses). Each frame in the sequence can be modeled via the EXM-HMT algorithm, while the sequence can be modeled based on the extension of previous HMM work [3]. 


\section{Figure Captions}

Figure 1. In the EXM-HMT classifier $P_{m}$ characteristic regions (subcomponents) are defined for each target class, an example of which is shown here. (a) Region corresponding to the overall image, (b) regions of the $P_{m}-1$ subcomponents (SCs).

Figure 2. Comparison of EXM and matched filters for the image in Fig. 1(a). (a) EXM filter of the image in Fig. 1(a), (b) matched Filter of the image in Fig. 1(a), (c) correlation of the EXM filter with the image in Fig. 1(a), (d) correlation of the matched filter with the image in Fig. 1(a).

Figure 3. The Karhunen-Loeve transform is used to reduce the training imagery to a set of eigen templates, with this performed for the overall target image and for the image subcomponents. Energy extracted by the eigenvectors for target subcomponent one (SC1 in Fig. 1), from 243 training images. The eigenvectors are arranged in a descending order of the corresponding eigenvalues; the top fifty eigenvectors extract $90 \%$ of the energy in the EXM filter set.

Figure 4. Neighborhoods used for $P_{m}$ regions in an associated target class (see (13)). The neighborhoods $(N s)$ are shown for the subcomponents of the target in Fig. 1, with a similar neighborhood defined to the overall image (Fig. 1(a)).

Figure 5. Example image and associated target subregions, as well as the associated hidden Markov tree (HMT). (a) Three-level HMT, with nodal index $p$ associated with component $\operatorname{corr}_{p}^{m, u}$ in the feature vector, (b) physical location of the image regions, as related to the HMT.

Figure 6. Schematization of the Radon-transform hidden-Markov-model (RT-HMM) algorithm. In the examples considered here the Radon transform of the image is obtained at angular intervals of $\delta \theta=30^{\circ}$.

Figure 7. Schematic of the levels of a wavelet transform. In the wavelet-HMT algorithm, the HMT is applied to a three-level quadtree, where each "parent" image (node) connected to four "child" nodes at a lower level.

Figure 8. Definition of the coordinate system employed, with target classes delineated.

Figure 9. Example FLIR images of class 1 and class 2 of the four vehicles under consideration. The blue regions correspond to low signal strength, with the strong regions 
represented in red. (a) Vehicle two, class one; (b) vehicle two, class two; (c) vehicle three, class one; (d) vehicle three, class two.

Figure 10. An angle-dependent symbol is used to delineate the number of FLIR image (at the associated target pose) for which the EXM-HMT algorithm misclassifies a given target class to the neighboring class, for the same vehicle (e.g. vehicle 1 class 1 is misclassified as either class 1 or 2 of vehicle 1). The radius corresponds to the number of misclassifications. Results are shown for vehicle one, with these representative of the results for all four targets.

Table 1. Confusion matrix for the wavelet-HMT algorithm. The front and back of the vehicle (classes one and three) and the two sides (classes two and four) are grouped together.

Table 2. Confusion matrix as in Table 1, for the RT-HMM algorithm.

Table 3. Confusion matrix as in Table 1, for the EXM-LVQ algorithm.

Table 4. Confusion matrix as in Table 1, for the EXM-HMT algorithm.

Table 5. Classification performance of the wavelet-HMT algorithms, defined with respect to identify the correct target (but not necessarily the correct target class).

Table 6. Confusion matrix as in Table 5, for the RT-HMM algorithm.

Table 7. Confusion matrix as in Table 5, for the EXM-LVQ algorithm.

Table 8. Confusion matrix as in Table 5, for the EXM-HMT algorithm. 


\section{References}

[1] D. Nandy and J. Ben-Arie, "Generalized feature extraction using expansion matching," IEEE Trans. on Image Processing, vol. 8, no. 1, pp. 22 -32, January 1999.

[2] M.S. Crouse, R.D. Novak and R.G. Baraniuk, "Wavelet-based statistical signal processing using hidden Markov models," IEEE Trans. Signal Processing, vol. 46, pp. 886-902, April 1998.

[3] N. Dasgupta, P. Runkle, L. Couchman and L. Carin, "Dual hidden Markov model for characterizing the wavelet coefficients from multi-aspect scattering data," submitted to IEEE Trans. Signal Processing.

[4] R. Kashyap and R. Chellapa, "Estimation and choice of neighbors in spatialinteraction models of images," IEEE Trans. on Information Theory, vol. IT-29, no. 1, pp. 60-72, January 1983.

[5] S. Geman and C. Graffigne, " Markov random field image models and their applications to computer vision," Proc. of the International Congress of Mathematics, pp. 1496-1517 1986.

[6] C. Nilubol, Q.H. Pham, R.M. Mersereau, M.J. T. Smith, and M.A. Clements, "Hidden Markov modeling for SAR automatic target recognition," Proc. of ICASSP98, vol. 1, pp.1061-1064, May 1998.

[7] A.M.P. Marinelli, L.M. Kaplan, N.M. Nasrabadi, "SAR ATR using a modified learning vector quantization algortihm," SPIE conference on Algorithms for SAR Imagery VI, pp. 343-354, April 1999.

[8] A.K. Jain, "Fundamentals of digital image processing," Prentice Hall, 1989.

[9] J. Ben-Arie, K. R. Rao, " A novel approach for template matching by nonorthogonal image expansion," IEEE Trans. on Circuits and Systems for Video Technology, vol. 3, no. 1, pp.71-84, February 1993.

[10] J. Li, A. Najmi and R.M. Gray, " Image classification by a two dimensional hidden Markov model," IEEE Transactions on Sig. Proc., vol. 48, no. 2, pp. 517-533, February 2000.

[11] L.R. Rabiner and B.H. Juang, “ An introduction to hidden Markov models,” IEEE Acoust. Speech Signal Proc. Magazine, vol. 3, pp. 4-16, Jan. 1986 
[12] L.R. Rabiner, "A tutorial on hidden Markov models and selected application in speech recognition," Proc. IEEE, vol. 77, pp. 257-285, Feb. 1989.

[13] P. R. Runkle, P. K. Bharadwaj, L. Couchman and L. Carin, “ Hidden Markov models for multi-aspect target classification," IEEE Trans. Signal Processing, vol. 47, no. 7, pp. 2035-2040, July 1999.

[14] P.K. Bharadwaj, P.R. Runkle and L. Carin, “ Target Identification with Wave-Based Matching Pursuits and Hidden Markov Model”, IEEE Trans. Antenna Propagation, vol.47, no. 10, pp. 1543-1554, Oct. 1999.

[15] T. Kohonen, "The self-organizing map," Proc. of the IEEE, vol. 78, no. 9, pp. 14641480 September 1990.

[16] Y. Linde, A. Buzo, and R. Gray, "An algorithm for vector quatization design," IEEE Trans. on Communications, vol. COM-28, no. 1, pp. 84-95, January 1980.

[17] Lin-Cheng Wang, Sandor Z. Der, and N.M. Nasrabadi, "Automatic target recognition using feature-decomposition and data-decomposition modular neural network", IEEE Trans. on Image Processing, vol. 7, no. 8, pp. 1113 -1121, August 1998. [18] M.J. Paulik and N. Mohankrishnan, " Shape recognition using a non-stationary autoregressive hidden Markov model," ICASSP, vol. 4, pp. 2377-2380, May 1991

[19] J. Deller, J. Proakis and J. Hansen, Discrete-Time Processing of Speech Signals, Englewood Cliffs, NJ: Prentice-Hall, 1993.

[20] S.E. Levinson, "Continuously variable duration hidden Markov model for automatic speech recognition," Computer Speech and Language, vol. 1, no.1, pp. 29-45, Mar. 1986. [21] X.Xie and R.J. Evans, “ Multiple target tracking and multiple frequency line tracking using hidden Markov models," IEEE Trans. Sig. Proc., vol. 39, pp. 2659-2676, 1991.

[22] F.S. Cohen, Z. Fan, and M.A. Patel, " classification of rotated and scaled textured images using Gaussian Markov random field models," IEEE Trans. on PAMI, vol. 13, no. 2, pp.192-201, February 1991.

[23] T.K. Moon, "The expectation maximization algorithm," IEEE Signal Processing Magazine, pp. 47-60,November 1996. 
[24] M.L. Cooper and M.I. Miller, "Information measures for target recognition accommodating signature variability," IEEE Trans. on Information Theory, vol. 46, no. 5, pp. 1896 -1907, August 2000. 


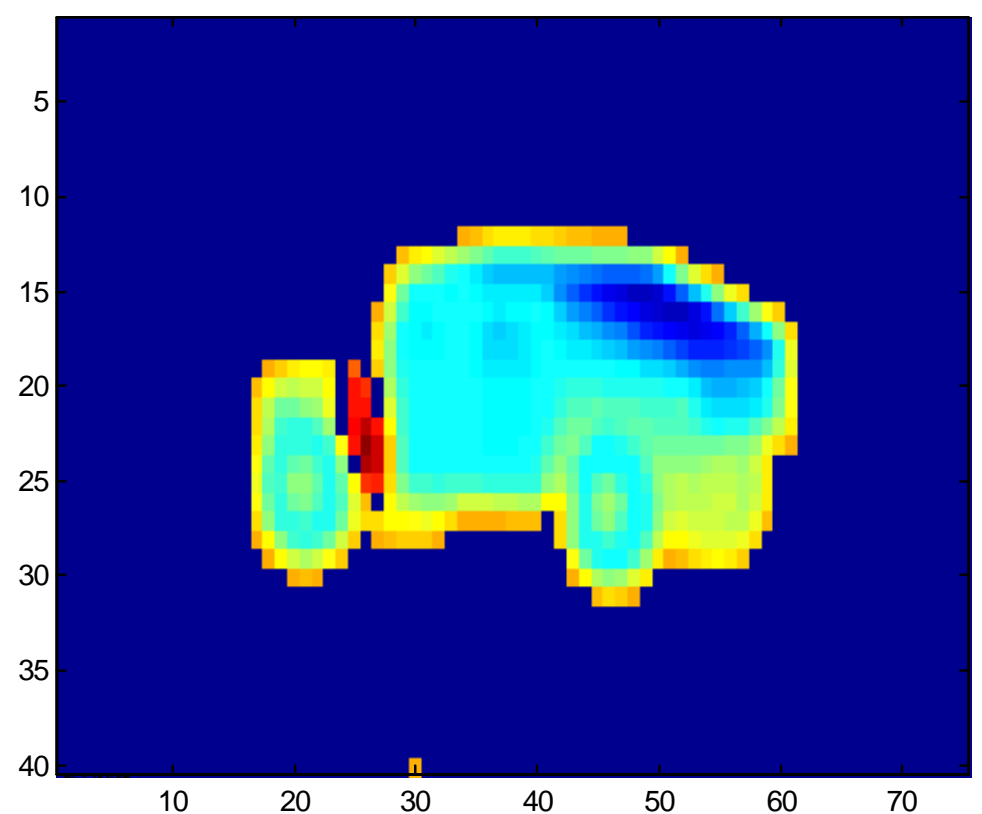

(a)

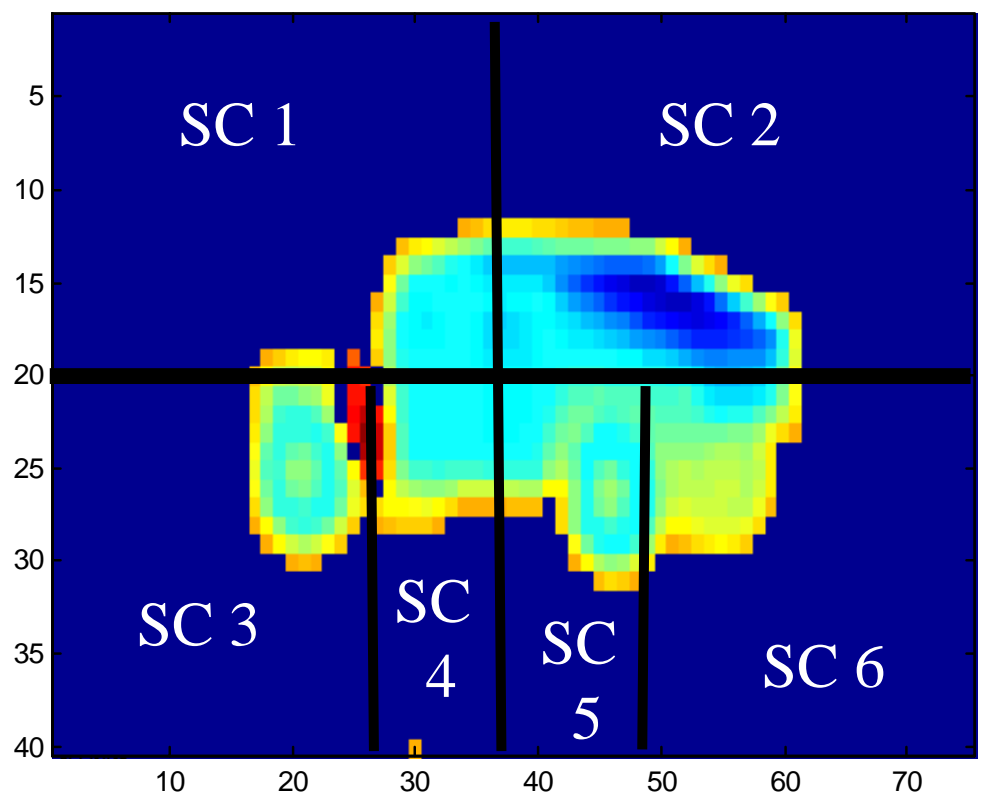

(b)

Figure 1 


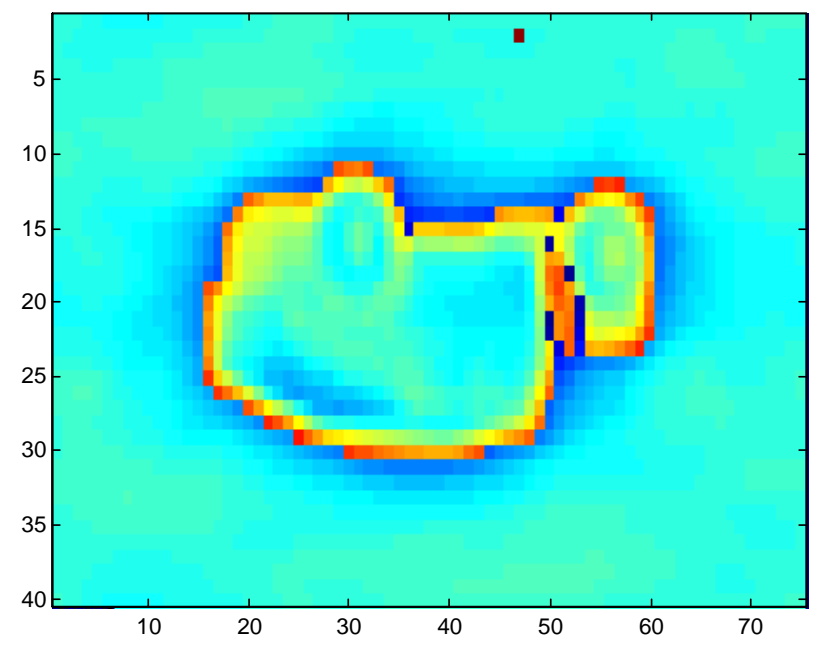

(a)

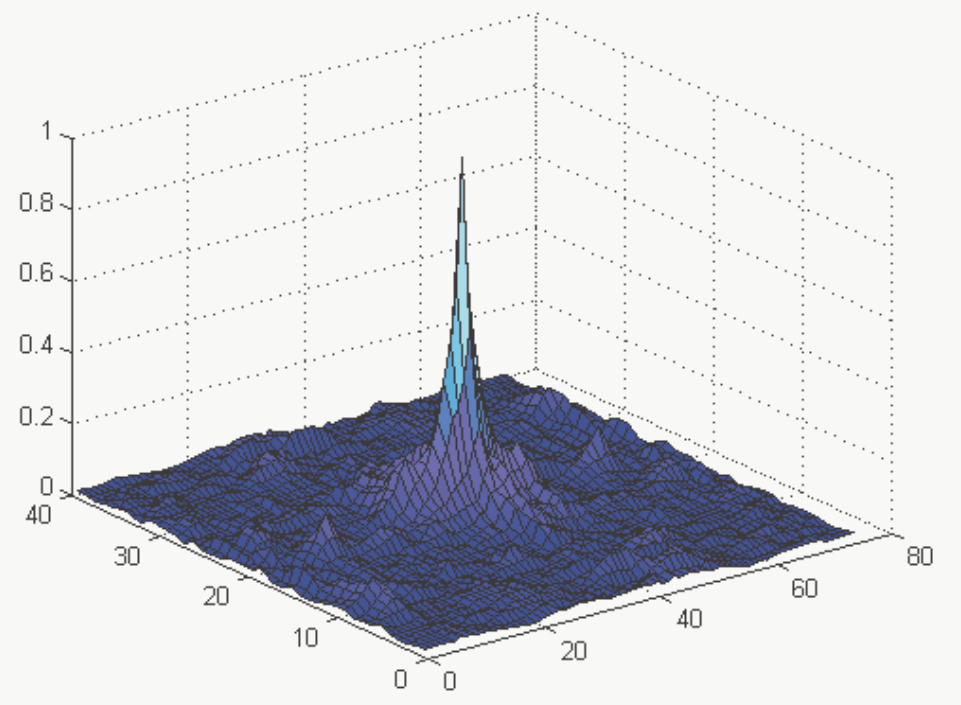

(c)

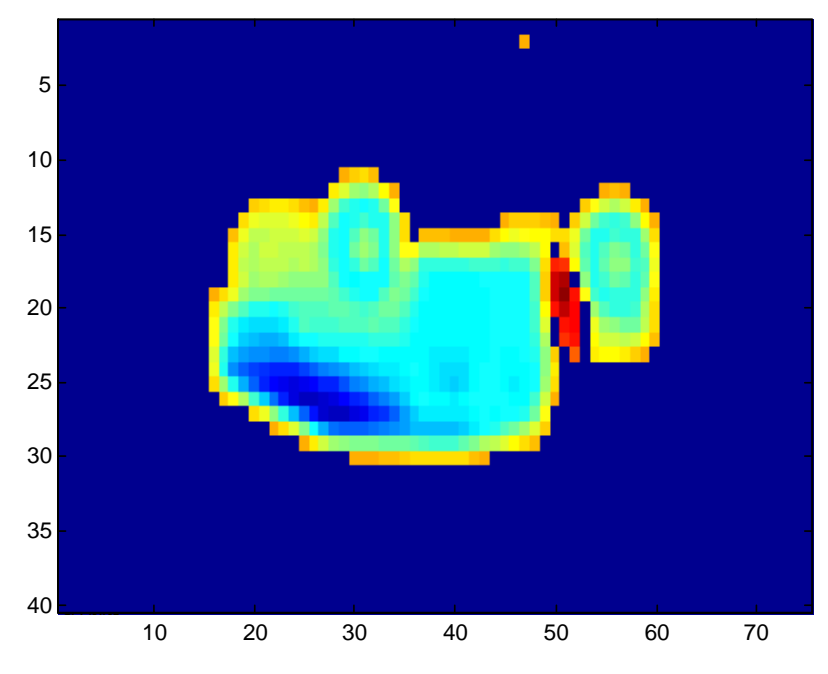

(b)

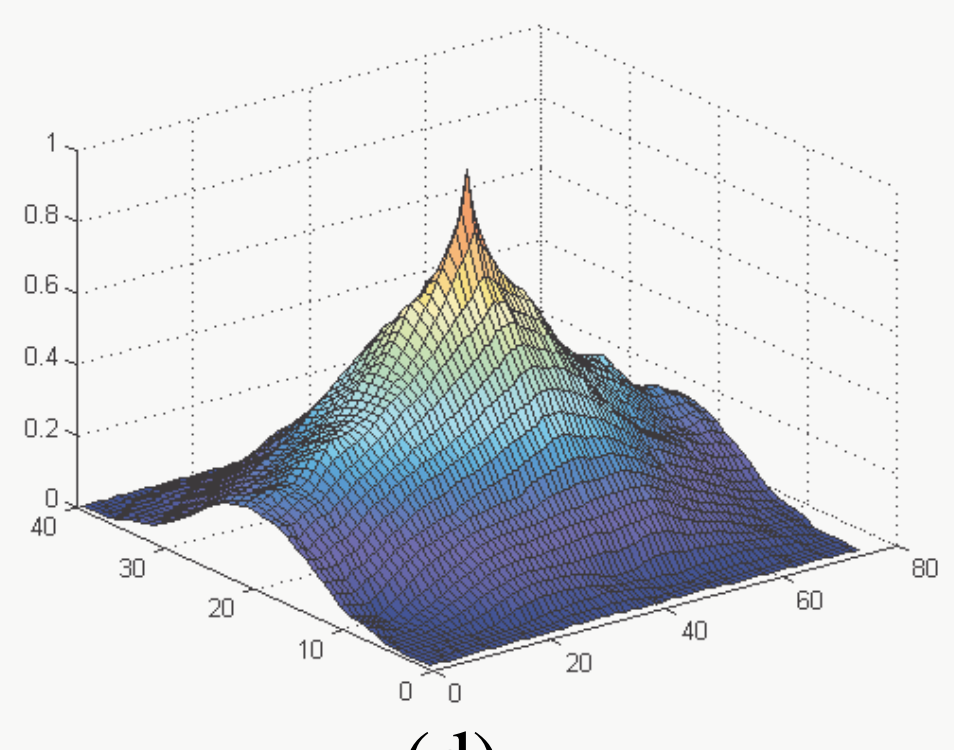

(d)

Figure 2 


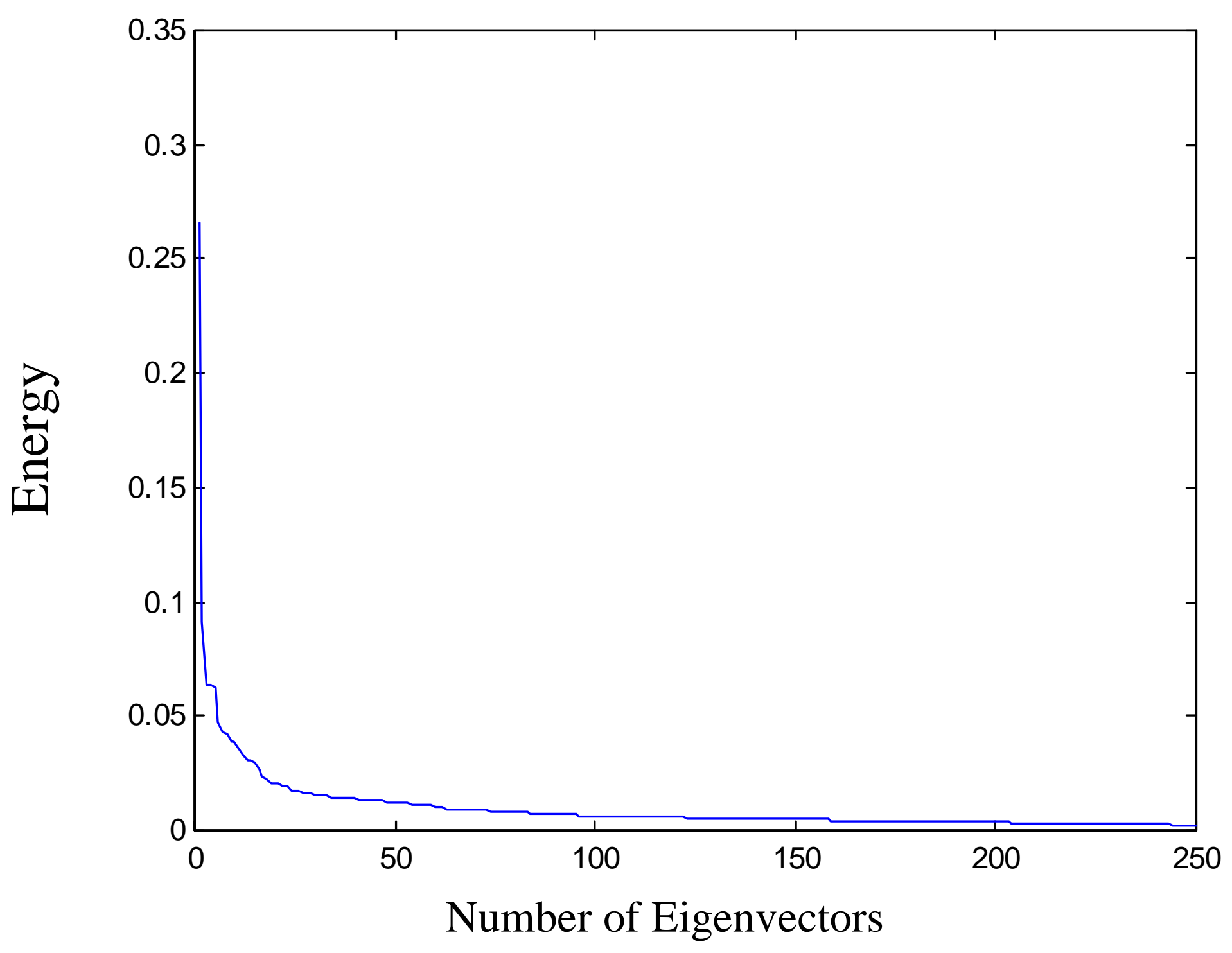

Figure 3 


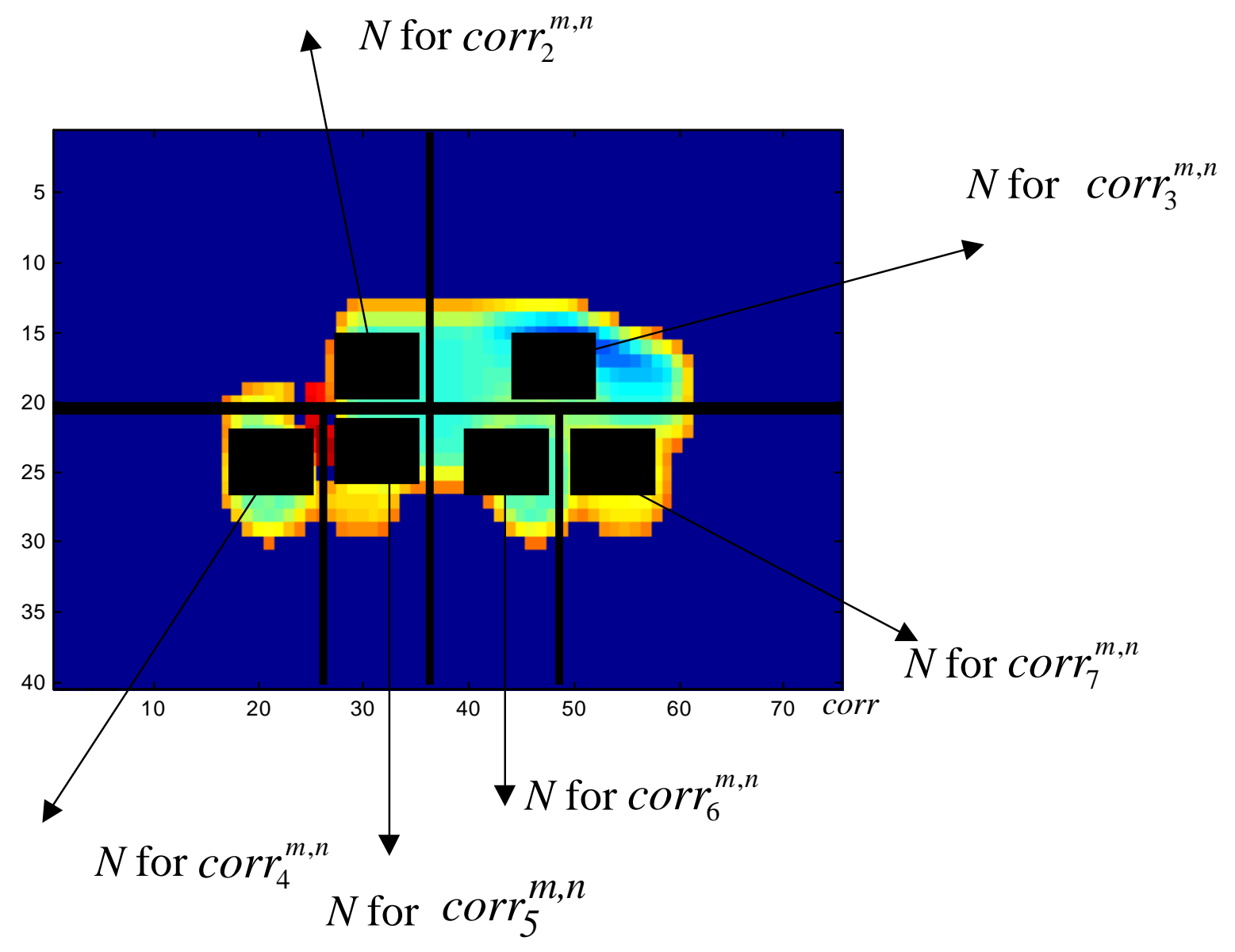

Figure 4 


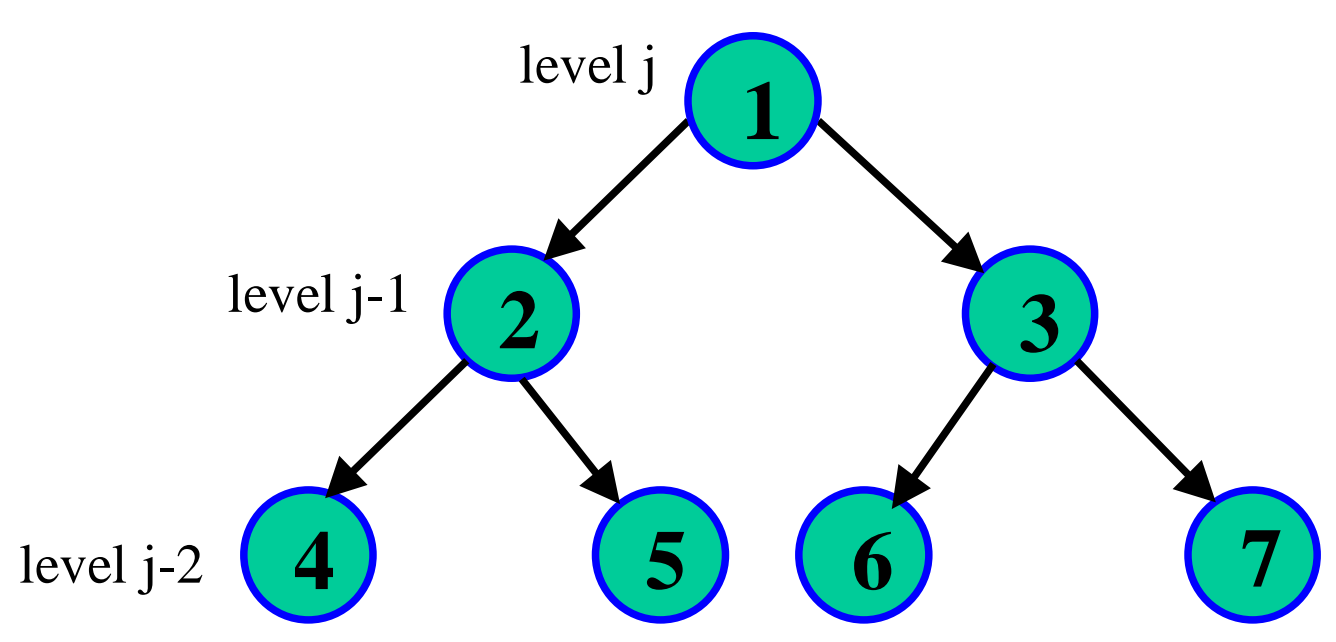

(a)

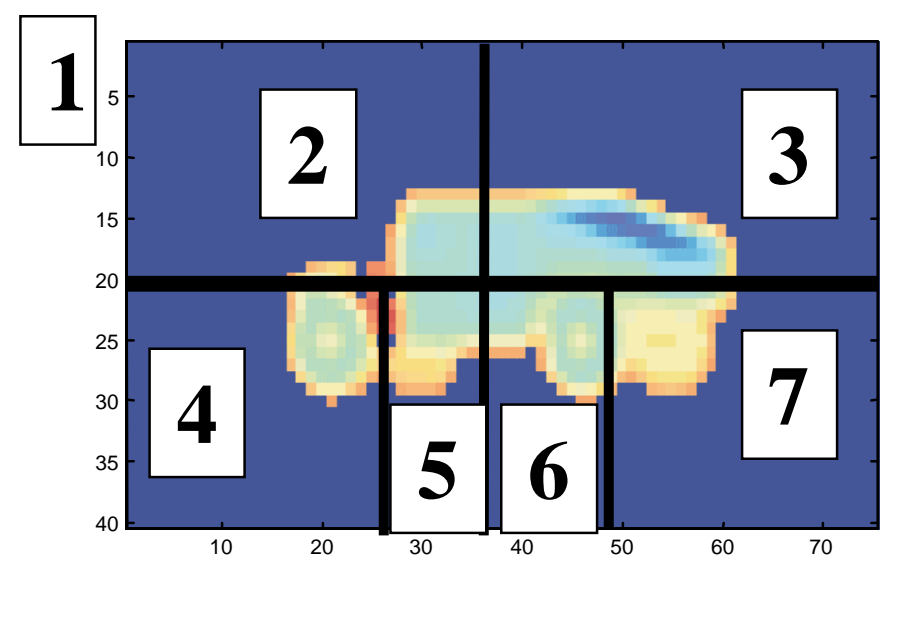

(b)

Figure 5 


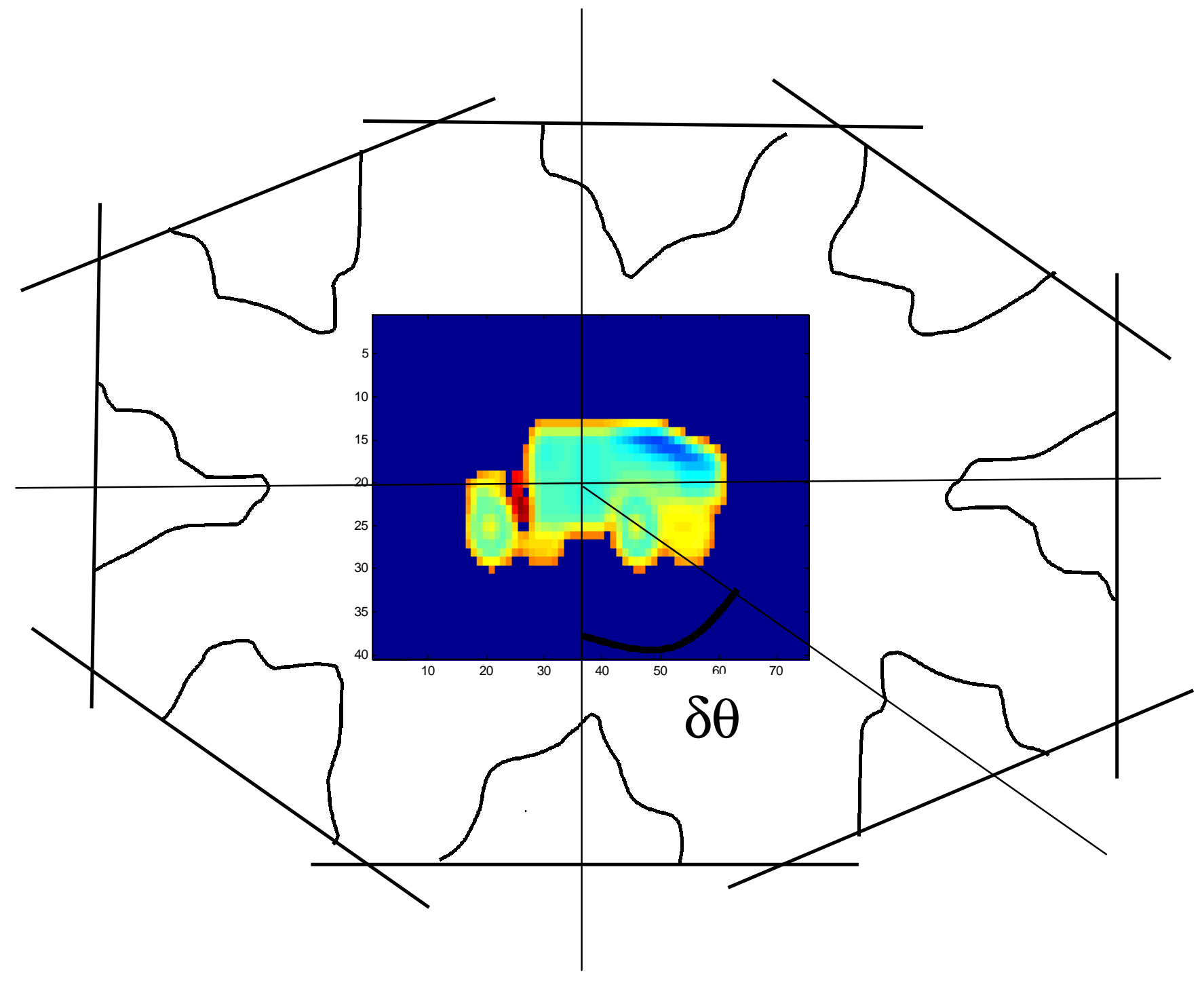

Figure 6 


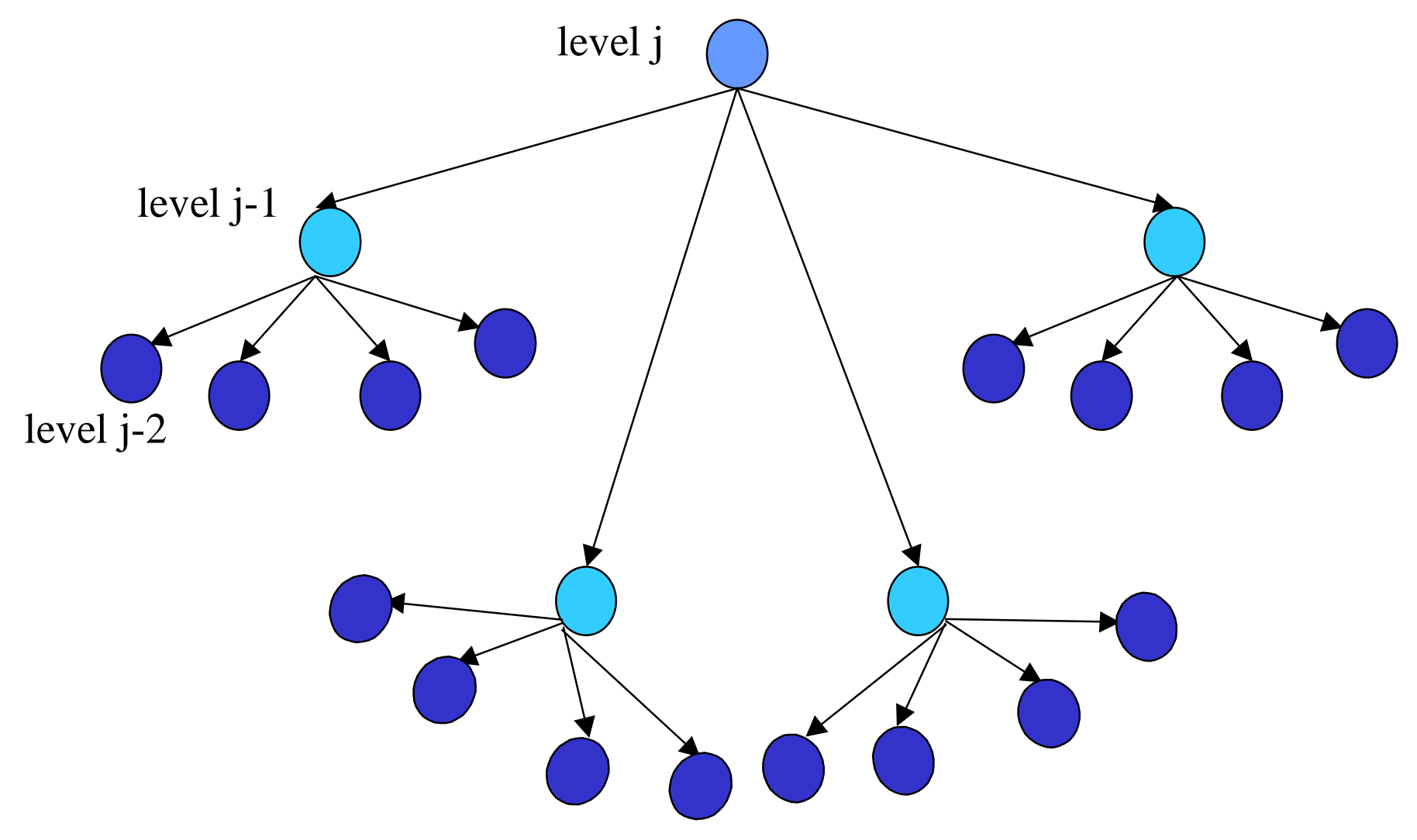

Figure 7 


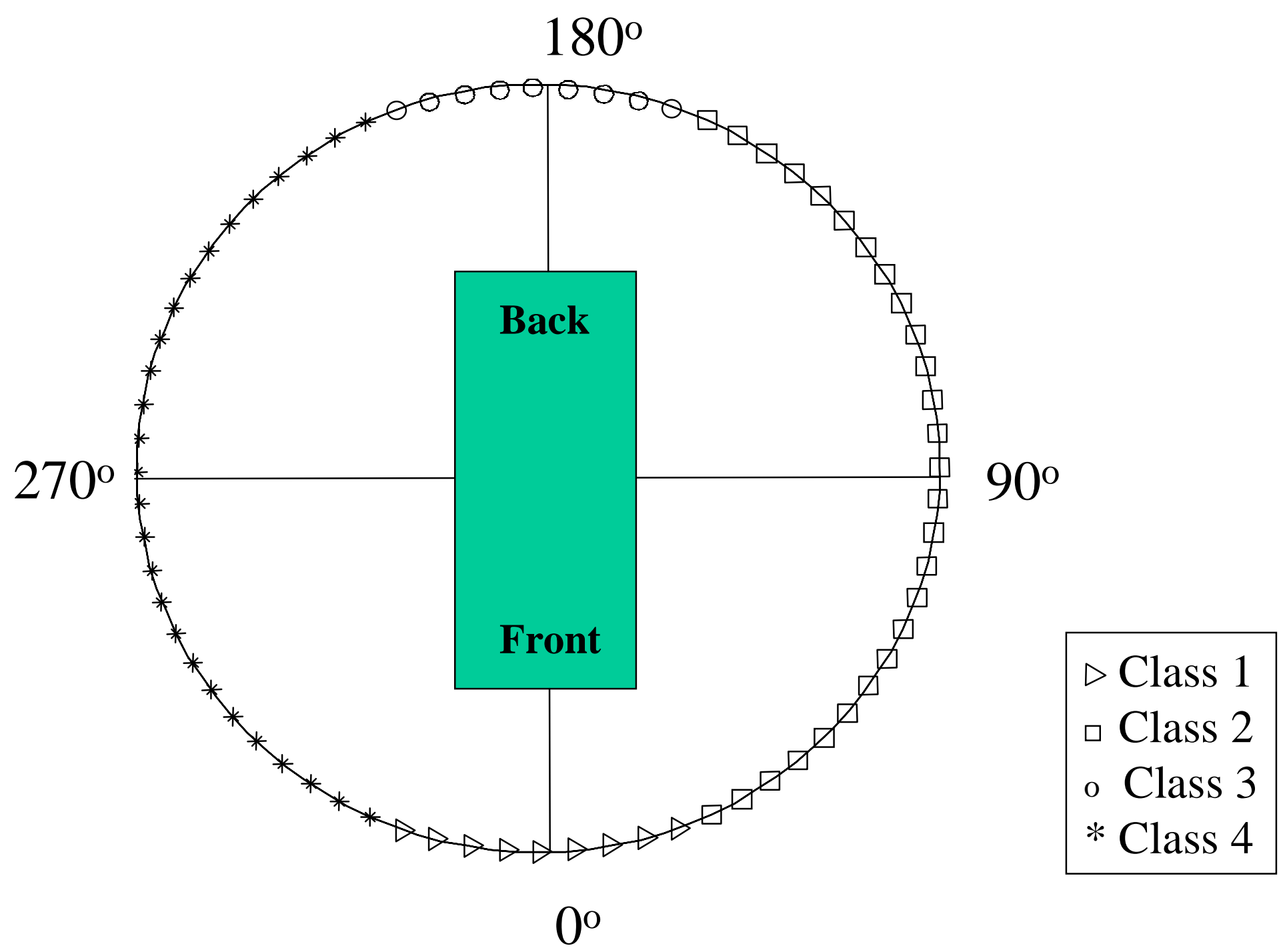

Figure 8 

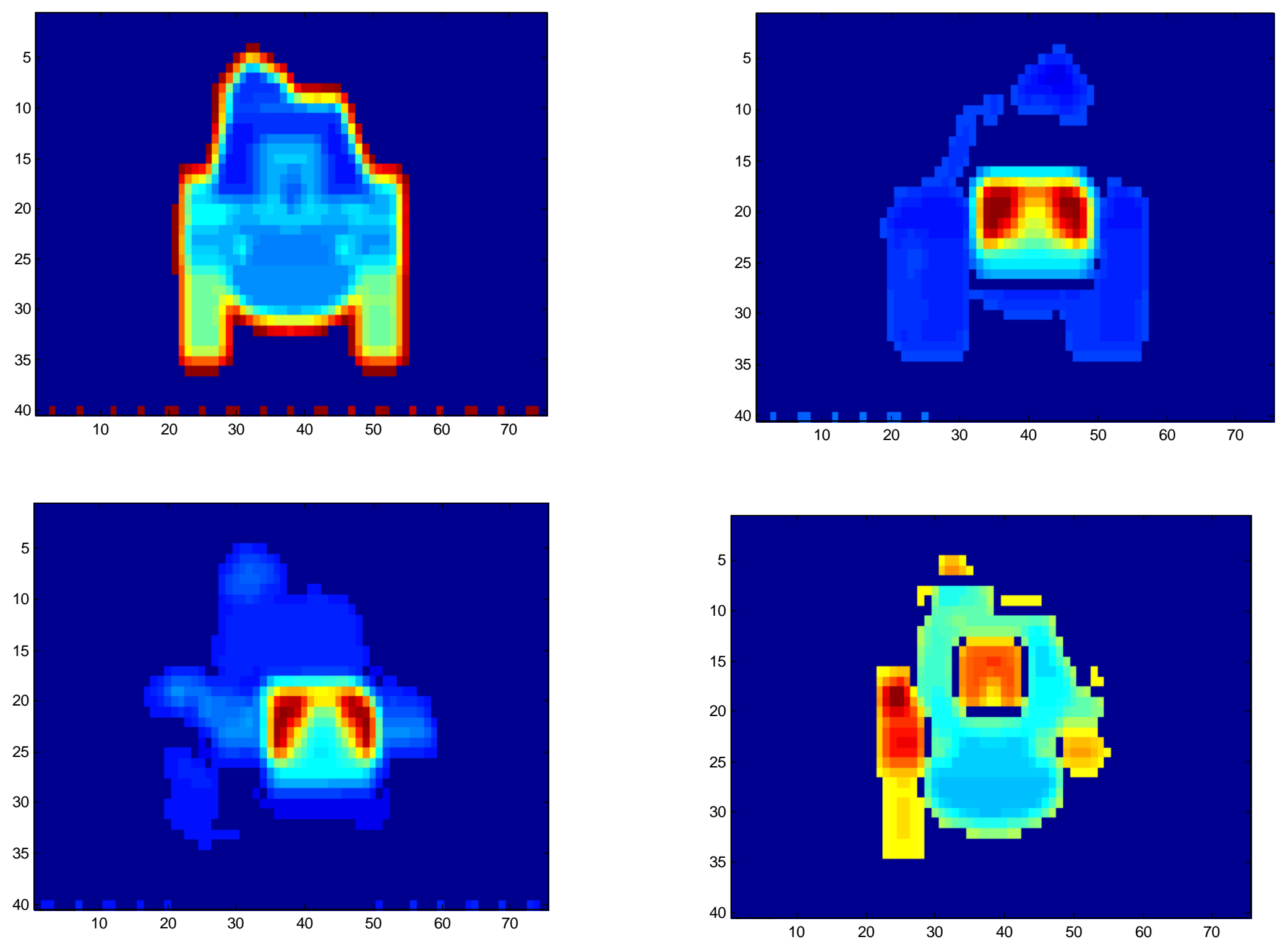

Figure 9(a) 

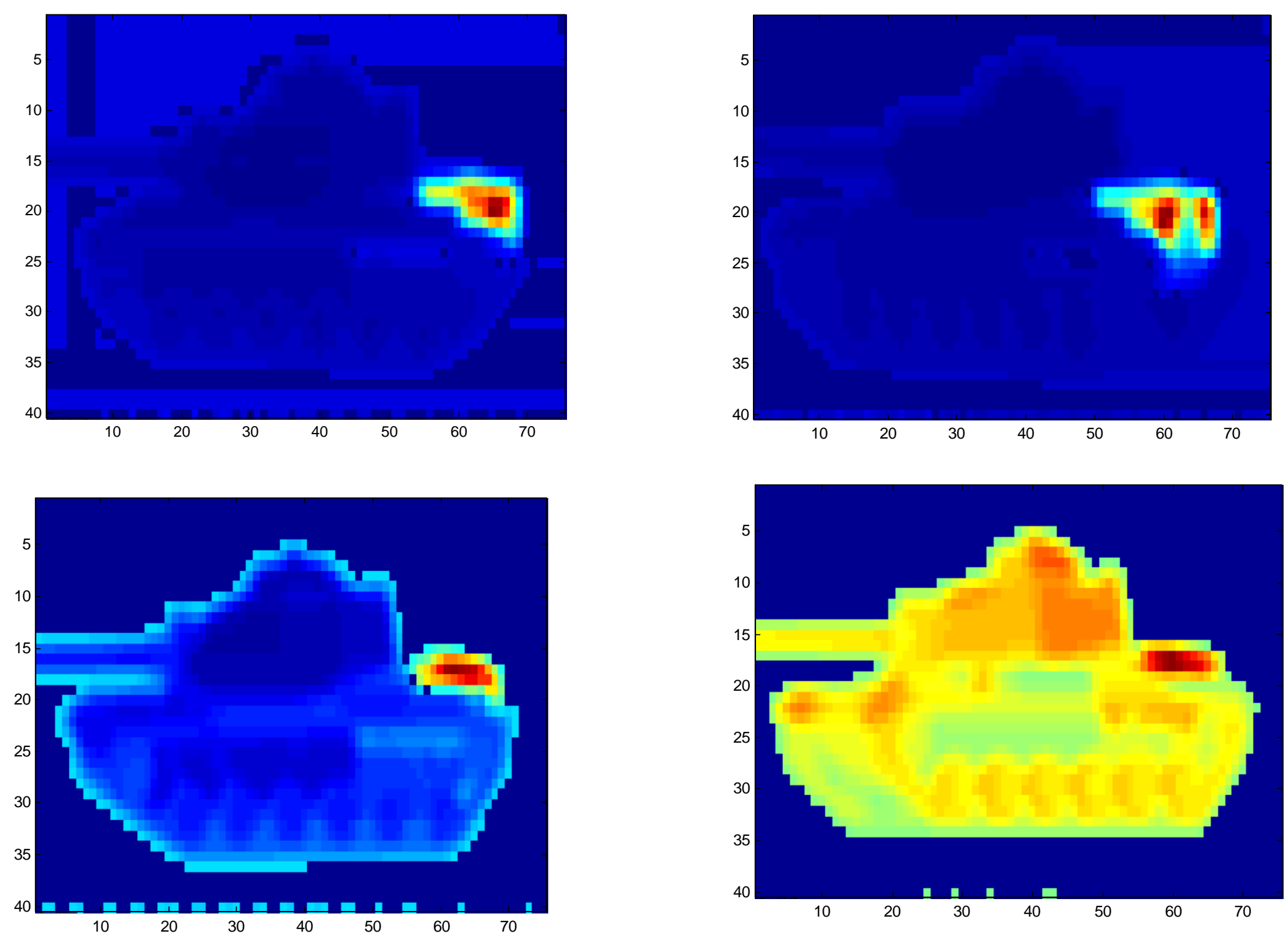

Figure 9(b) 

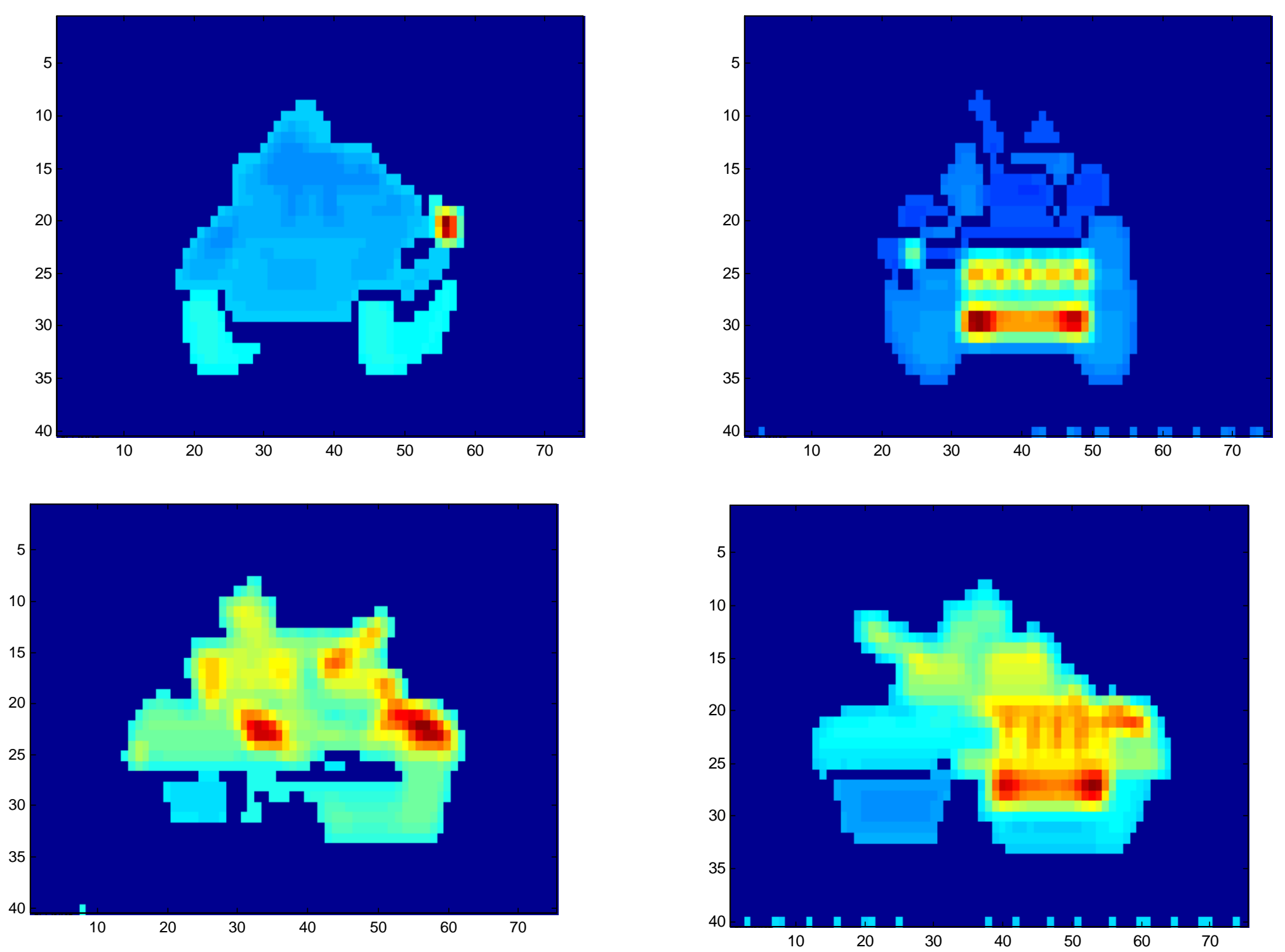

Figure 9(c) 

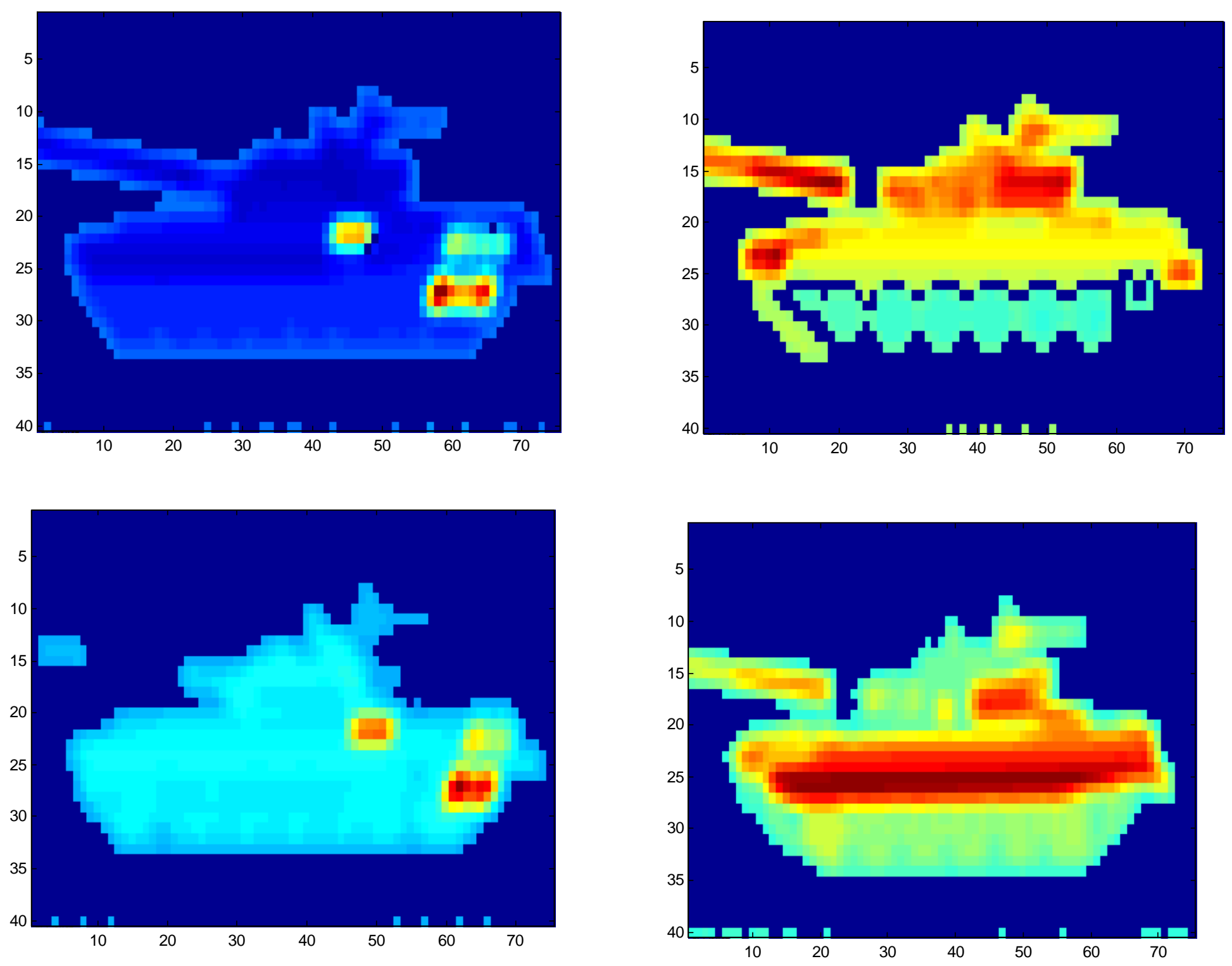

Figure 9(d) 


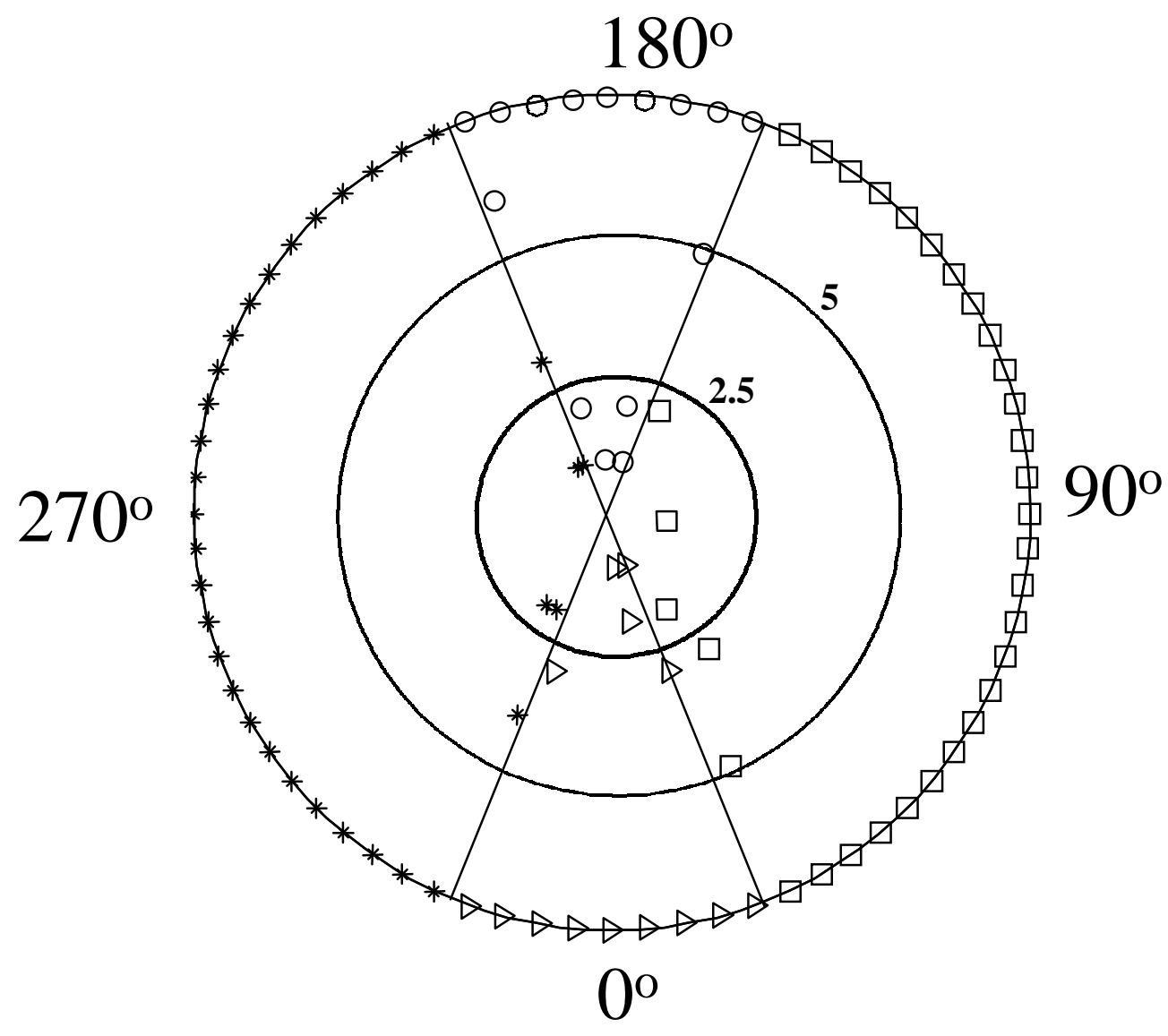

$\triangleright$ Class 1

$\square$ Class 2

- Class 3

* Class 4 


\begin{tabular}{|c|c|c|c|c|c|c|c|c|}
\hline & V1 $\quad C \quad 1 \& 3$ & V1 C 2\&4 & $\mathrm{V} 2 \mathrm{C} 1 \& 3$ & V2 C 2\&4 & V3 C $1 \& 3$ & V3 C $2 \& 4$ & V4 C $1 \& 3$ & V4 C 2\&4 \\
\hline $\begin{array}{l}\text { V1 C } \\
1 \& 3\end{array}$ & 56 & 4 & 6 & 0 & 9 & 0 & 25 & 0 \\
\hline $\begin{array}{l}\text { V1 C } \\
2 \& 4\end{array}$ & 5 & 78 & 3 & 1 & 3 & 0 & 6 & 4 \\
\hline $\begin{array}{l}\mathrm{V} 2 \mathrm{C} \\
1 \& 3\end{array}$ & 12 & 9 & 48 & 2 & 13 & 2 & 6 & 8 \\
\hline $\begin{array}{l}\text { V2C } \\
2 \& 4\end{array}$ & 0 & 1 & 9 & 73 & 2 & 8 & 0 & 7 \\
\hline $\begin{array}{l}\mathrm{V} 3 \mathrm{C} \\
1 \& 3\end{array}$ & 7 & 9 & 9 & 0 & 48 & 0 & 17 & 10 \\
\hline $\begin{array}{l}\text { V3 C } \\
2 \& 4\end{array}$ & 0 & 1 & 1 & 6 & 0 & 79 & 0 & 11 \\
\hline $\begin{array}{l}\mathrm{V} 4 \mathrm{C} \\
1 \& 3\end{array}$ & 20 & 12 & 3 & 0 & 17 & 0 & 44 & 3 \\
\hline $\begin{array}{l}\text { V4 C } \\
2 \& 4\end{array}$ & 1 & 15 & 14 & 0 & 10 & 2 & 3 & 54 \\
\hline
\end{tabular}

Table 1 


\begin{tabular}{|c|c|c|c|c|c|c|c|c|}
\hline & V1 C $1 \& 3$ & V1 C $2 \& 4$ & V2 C $1 \& 3$ & V2 C $2 \& 4$ & V3 C $1 \& 3$ & V3 C $2 \& 4$ & V4 C $1 \& 3$ & V4 C $2 \& 4$ \\
\hline $\begin{array}{l}\text { V1 C } \\
1 \& 3\end{array}$ & 67 & 7 & 8 & 0 & 1 & 0 & 17 & 1 \\
\hline $\begin{array}{l}\mathrm{V} 1 \mathrm{C} \\
2 \& 4\end{array}$ & 4 & 79 & 2 & 1 & 2 & 2 & 3 & 6 \\
\hline $\begin{array}{l}\mathrm{V} 2 \mathrm{C} \\
1 \& 3\end{array}$ & 1 & 3 & 57 & 9 & 10 & 1 & 11 & 8 \\
\hline $\begin{array}{l}\text { V2 C } \\
2 \& 4\end{array}$ & 0 & 0 & 1 & 56 & 1 & 36 & 0 & 6 \\
\hline $\begin{array}{l}\text { V3 C } \\
1 \& 3\end{array}$ & 1 & 6 & 8 & 1 & 50 & 0 & 19 & 16 \\
\hline $\begin{array}{l}\text { V3 C } \\
2 \& 4\end{array}$ & 0 & 0 & 0 & 10 & 1 & 85 & 0 & 4 \\
\hline $\begin{array}{l}\text { V4 C } \\
1 \& 3\end{array}$ & 15 & 0 & 11 & 1 & 15 & 0 & 52 & 6 \\
\hline $\begin{array}{l}\mathrm{V} 4 \mathrm{C} \\
2 \& 4\end{array}$ & 0 & 6 & 7 & 5 & 16 & 2 & 3 & 62 \\
\hline
\end{tabular}

Table 2 


\begin{tabular}{|c|c|c|c|c|c|c|c|c|}
\hline & V1 C $1 \& 3$ & V1 C 2\&4 & $\mathrm{V} 2 \mathrm{C} 1 \& 3$ & V2 C 2\&4 & V3 C 1\&3 & $\mathrm{V} 3 \mathrm{C} 2 \& 4$ & $\overline{V 4 C ~} 1 \& 3$ & $\mathrm{~V} 4 \mathrm{C} 2 \& 4$ \\
\hline $\begin{array}{l}\text { V1 C } \\
1 \& 3\end{array}$ & 73 & 14 & 3 & 0 & 0 & 0 & 9 & 1 \\
\hline $\begin{array}{l}\text { V1 C } \\
2 \& 4\end{array}$ & 15 & 71 & 2 & 0 & 2 & 1 & 1 & 6 \\
\hline $\begin{array}{l}\mathrm{V} 2 \mathrm{C} \\
1 \& 3\end{array}$ & 12 & 3 & 56 & 9 & 9 & 2 & 5 & 3 \\
\hline $\begin{array}{l}\text { V2 C } \\
2 \& 4\end{array}$ & 3 & 0 & 5 & 81 & 1 & 9 & 1 & 1 \\
\hline $\begin{array}{l}\text { V3 C } \\
1 \& 3\end{array}$ & 9 & 4 & 10 & 2 & 64 & 6 & 1 & 4 \\
\hline $\begin{array}{l}\text { V3 C } \\
2 \& 4\end{array}$ & 1 & 1 & 0 & 9 & 3 & 84 & 0 & 2 \\
\hline $\begin{array}{l}\text { V4 C } \\
1 \& 3\end{array}$ & 16 & 0 & 3 & 0 & 5 & 0 & 66 & 10 \\
\hline $\begin{array}{l}\text { V4 C } \\
2 \& 4\end{array}$ & 2 & 2 & 1 & 4 & 2 & 5 & 1 & 82 \\
\hline
\end{tabular}

Table 3 


\begin{tabular}{|c|c|c|c|c|c|c|c|c|}
\hline & V1 C 1\&3 & $\overline{V 1 C ~} 2 \& 4$ & $\mathrm{~V} 2 \mathrm{C} 1 \& 3$ & $\mathrm{~V} 2 \mathrm{C} 2 \& 4$ & V3 C 1\&3 & V3 C 2\&4 & $\overline{V 4}$ C $1 \& 3$ & $\mathrm{~V} 4 \mathrm{C} 2 \& 4$ \\
\hline $\begin{array}{l}\text { V1 C } \\
1 \& 3\end{array}$ & 82 & 15 & 1 & 0 & 0 & 0 & 2 & 0 \\
\hline $\begin{array}{l}\text { V1 C } \\
2 \& 4\end{array}$ & 5 & 90 & 1 & 0 & 1 & 0 & 0 & 3 \\
\hline $\begin{array}{l}\mathrm{V} 2 \mathrm{C} \\
1 \& 3\end{array}$ & 6 & 2 & 71 & 9 & 4 & 1 & 1 & 6 \\
\hline $\begin{array}{l}\text { V2 C } \\
2 \& 4\end{array}$ & 0 & 0 & 4 & 89 & 0 & 3 & 1 & 2 \\
\hline $\begin{array}{l}\text { V3 C } \\
1 \& 3\end{array}$ & 6 & 2 & 6 & 0 & 81 & 3 & 1 & 2 \\
\hline $\begin{array}{l}\text { V3 C } \\
2 \& 4\end{array}$ & 0 & 0 & 0 & 7 & 3 & 87 & 0 & 2 \\
\hline $\begin{array}{l}\text { V4 C } \\
1 \& 3\end{array}$ & 9 & 0 & 2 & 0 & 2 & 0 & 78 & 9 \\
\hline $\begin{array}{l}\text { V4 C } \\
2 \& 4\end{array}$ & 1 & 1 & 3 & 2 & 4 & 2 & 1 & 86 \\
\hline
\end{tabular}

Table 4 


\begin{tabular}{|c|c|c|c|c|}
\hline & Vehicle 1 & Vehicle 2 & Vehicle 3 & Vehicle 4 \\
\hline Vehicle 1 & 77 & 4 & 5 & 14 \\
\hline Vehicle 2 & 6 & 74 & 11 & 9 \\
\hline Vehicle 3 & 5 & 8 & 72 & 15 \\
\hline Vehicle 4 & 20 & 11 & 14 & 55 \\
& & 11 & & \\
\hline
\end{tabular}

Table 5 


\begin{tabular}{|c|c|c|c|c|}
\hline & Vehicle 1 & Vehicle 2 & Vehicle 3 & Vehicle 4 \\
\hline Vehicle 1 & 81 & 3 & 3 & 11 \\
\hline Vehicle 2 & 1 & 59 & 31 & 9 \\
\hline Vehicle 3 & 2 & 9 & 77 & 12 \\
\hline Vehicle 4 & 8 & 12 & 17 & 63 \\
\hline
\end{tabular}

Table 6 


\begin{tabular}{|c|c|c|c|c|}
\hline & Vehicle 1 & Vehicle 2 & Vehicle 3 & Vehicle 4 \\
\hline Vehicle 1 & 86 & 3 & 3 & 8 \\
\hline Vehicle 2 & 6 & 81 & 10 & 3 \\
\hline Vehicle 3 & 5 & 10 & 82 & 3 \\
\hline Vehicle 4 & 7 & 5 & 7 & 82 \\
\hline
\end{tabular}

Table 7 


\begin{tabular}{|c|c|c|c|c|}
\hline & Vehicle 1 & Vehicle 2 & Vehicle 3 & Vehicle 4 \\
\hline Vehicle 1 & 96 & 1 & 1 & 2 \\
\hline Vehicle 2 & 2 & 90 & 4 & 3 \\
\hline Vehicle 3 & 2 & 7 & 88 & 2 \\
\hline Vehicle 4 & 4 & 4 & 5 & 87 \\
\hline
\end{tabular}

Table 8 